\title{
Noncommutative Geometry of Phase Space
}

\author{
Zakaria Giunashvili
}

October 30, 2018

\begin{abstract}
We investigate the geometric, algebraic and homologic structures related with Poisson structure on a smooth manifold. Introduce a noncommutative foundations of these structures for a Poisson algebra. Introduce and investigate noncommutative Bott connection on a foliated manifold using the algebraic definition of submanifold and quotient manifold. Develop an algebraic construction for the reduction of a degenerated Poisson algebra.
\end{abstract}




\section{Contents}

1 Distributions on $\mathbf{C}^{\infty}$ - Class Manifolds: General Overview 3

2 Derivation Based Noncommutative Differential Calculus $\quad 10$

2.1 Noncommutative Differential Forms. . . . . . . . . . . . . . . 10

2.2 Noncommutative Submanifold. . . . . . . . . . . . . . . . . . . 11

2.3 Noncommutative Quotient Manifold. . . . . . . . . . . . . . . . 12

2.4 Noncommutative Connection and Curvature. . . . . . . . . . . 13

2.5 Connection Compatible with Group Action. . . . . . . . . . . 14

2.6 Noncommutative Distribution. Integral Manifold and Bott Con-

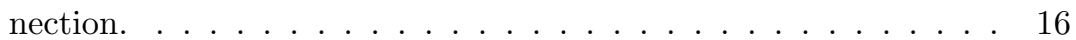

3 Lie Superalgebra Structures on the Space of Multiderivations 21

3.1 Compositional Product and Supercommutator. . . . . . . . . 21

3.2 Supercommutator on the Space of Multiderivations . . . . . . . . 22

3.3 Poisson Structure. Poisson Cohomologies . . . . . . . . . . . . . 23

3.4 External Differential as a Supercommutator . . . . . . . . . . . . 24

4 Schouten Bracket as the Deviation of the Coboundary Operator from the Leibnitz Rule 29

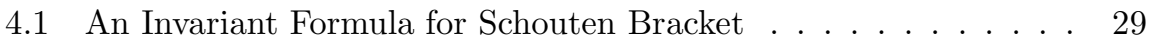

4.2 Star Operator for a Poisson Structure. Poisson Cohomologies. . . 31

5 Differential Complex and Generalized Functions on Poisson Manifold 35

5.1 Brief Overview of Geometric structures on Poisson Manifold . . . 35

5.2 Brief Review of Distributions (Generalized Functions) on Smooth

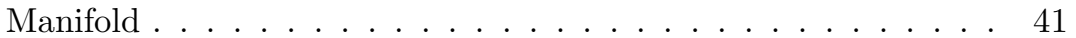

5.3 Poisson Bracket on Generalized Functions and Generalized Casimin

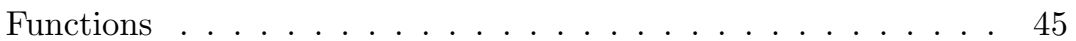

5.4 The Canonical Comlex of a Poisson Manifold and Generalized Casimir Functions . . . . . . . . . . . . . . . 47

5.5 Poisson Ideal and Reduction of Poisson Algebra. . . . . . . . . . 50 


\section{Distributions on $\mathrm{C}^{\infty}$ - Class Manifolds: Gen- eral Overview}

In this section we give a brief overview of some definitions and facts concerning the distributions on $\mathrm{C}^{\infty}$ - class manifold. We consider not only regular distributions (i.e., the distributions with a constant rank), but singular distributions too (i.e., the distributions the rank of which varies from point to point).

For any vector space $V$ and $k \in \mathbf{N}$, we denote by $\operatorname{Gr}_{k}(V)$ the Grassmann manifold of $k$-dimensional vector subspaces of the vector space $V$.

Definition 1 Let $M$ be a $C^{\infty}$ - class manifold and $\pi: E \longrightarrow M$ be a vector fiber bundle. The fiber bundle $\widetilde{\pi}_{k}: G r_{k}(E) \longrightarrow M$ is called the Grassmanization of the fiber bundle $\pi: E \longrightarrow M$, or the Grassmanian fiber bundle corresponding to $\pi: E \longrightarrow M$, if for each point $x_{0} \in M$ its fiber $\widetilde{\pi}^{-1}\left(x_{0}\right)$ is the Grassmann manifold of $k$-dimensional subspaces of the vector space $\pi^{-1}\left(x_{0}\right)$.

Let $\tau: \mathrm{T}(M) \longrightarrow M$ be the tangent vector bundle over the manifold $M$.

Definition 2 For an integer number $k$ such that $1 \leq k \leq n$, where $n=$ $\operatorname{dim}(M)$, a $k$-dimensional distribution $D$, on the manifold $M$, is a correspondence $x \mapsto D(x)$, where $x \in M$ and $D(x)$ is a $k$-dimensional subspace of the tangent space $T_{x}(M)$.

Consider the Grassmanization of the tangent bundle over the manifold $M$

$$
\widetilde{\tau}_{k}: G r_{k}(T(M)) \longrightarrow M
$$

A $k$-dimensional distribution $D$, can be considered as a section of this fiber bundle:

$$
M \ni x \mapsto D(x) \in G r_{k}\left(T_{x}(M)\right)
$$

The distribution $D$ is said to be a $C^{\infty}$ - class, or smooth distribution, if $D$ is a $C^{\infty}$ - class section.

Definition 3 A smooth distribution $D$ is called involutive, if for any two smooth vector fields $X$ and $Y$ on the manifold $M$, such that for each $x \in M$ the vectors $X(x)$ and $Y(x)$ are elements of the vector space $D(x)$, their commutator $[X, Y]$ is also such that for each $x \in M$ the vector $[X, Y](x)$ is an element of the vector space $D(x)$.

Definition 4 A submanifold $N$ of the manifold $M$ is said to be an integral submanifold for a given distribution $D$ on $M$ if for each point $x \in N$, we have that $T_{x}(N)=D(x)$.

$A$ distribution $D$ on the manifold $M$ is said to be integrable if for each point $x \in M$ there exists an integral submanifold $N$ for the distribution $D$, such that $x \in N$. 
The Frobenius' classical theorem states that, a $\mathrm{C}^{\infty}$ - class distribution $D$ on a $\mathrm{C}^{\infty}$ - class manifold is integrable if and only if $D$ is involutive. Moreover, if the distribution $D$ is involutive, then for each point $x_{0} \in M$, there exists its neighborhood $U$ and a coordinate system

$$
u_{1}, u_{2}, \ldots, u_{n}: U \longrightarrow \mathbf{R}
$$

such that the level submanifolds

$$
u_{i}=\text { const }, \quad i=k+1, \ldots, n
$$

are the integral submanifolds of the distribution $D$. Moreover: if $N$ is a connected integral submanifold, such that $N \subset U$, then $N$ is inside of one of these level submanifolds.

E. Cartan's formalism gives a different approach to the local properties of distributions. This formalism is more general, and is designed for studying the geometric properties of not only distributions, but also higher-order differential equations (see, for example [26] and 37]).

Definition 5 (Cartan distribution) For any point $V \in G r_{k}(T(M))$, let

$$
K_{V}=\left(\left(\widetilde{\tau}_{k}\right)^{\prime}(V)\right)^{-1}(V)
$$

be the subspace of the vector space $T_{V}\left(G r_{k}(T(M))\right)$ where

$$
\left(\widetilde{\tau}_{k}\right)^{\prime}(V): T_{V}\left(G r_{K}(T(M))\right) \longrightarrow T_{x}(M)
$$

is the differential of the projection mapping

$$
\widetilde{\tau}_{k}: G r_{k}(T(M)) \longrightarrow M
$$

at the point $V$, and $x=\widetilde{\tau}_{k}(V)$.

The distribution

$$
G r_{k}(T(M)) \ni V \mapsto K_{V} \subset T_{V}\left(G r_{k}(T(M))\right)
$$

is called the Cartan distribution on the Graasmanization of the tangent bundle $T(M)$.

As the fiber of the fiber bundle $\widetilde{\tau}_{k}: \operatorname{Gr}_{k}(\mathrm{~T}(M)) \longrightarrow M$ is the Grassmann manifold with dimension equal to $k(n-k)$, the dimension of the total space $\mathrm{Gr}_{k}(\mathrm{~T}(M))$ is equal to $n+k(n-k)$

Lemma 1 For each $W \in G r_{k}(T(M))$ the dimension of the subspace $K_{W}$, correspondent to the Cartan distribution at the point $W$ is equal to $k(n-k+1)$.

Proof. Let $V_{W}$ be the vertical tangent space of the Grassmannian fiber bundle

$$
\widetilde{\tau}_{k}: \operatorname{Gr}_{k}(\mathrm{~T}(M)) \longrightarrow M
$$


at the point $W \in \operatorname{Gr}_{k}\left(\mathrm{~T}_{x}(M)\right)$. We have the following exact sequence

$$
0 \longrightarrow V_{W} \stackrel{\imath}{\hookrightarrow} K_{W} \stackrel{\left(\tilde{\tau}_{k}\right)^{\prime}(W)}{\longrightarrow} W \longrightarrow 0
$$

from which follows that $\operatorname{dim}\left(K_{W}\right)=\operatorname{dim}\left(V_{W}\right)+\operatorname{dim}(W)$. The space $V_{W}$ is the tangent space of the Grassmann manifold $\operatorname{Gr}_{k}\left(\mathrm{~T}_{x}(M)\right)$ at the point $W$, and as it is well-known, is isomorphic to the space $\operatorname{Hom}\left(W, \mathrm{~T}_{x}(M) / W\right)$. Therefore, we have that

$$
\operatorname{dim}\left(K_{W}\right)=\operatorname{dim}\left(H o m\left(W, \mathrm{~T}_{x}(M) / W\right)\right)+\operatorname{dim}(W)=k(n-k+1)
$$

Let $X, Y$ and $Z$ be finite-dimensional real vector spaces and the following is an exact sequence of linear mappings

$$
0 \longrightarrow \operatorname{Hom}(Z, X) \stackrel{\imath}{\hookrightarrow} Y \stackrel{\pi}{\longrightarrow} Z \longrightarrow 0
$$

Any splitting, $s: Z \longrightarrow Y$, of this exact sequence defines an antisymmetric, bilinear form $\langle\cdot, \cdot\rangle_{s}$ on the vector space $Y$, which takes its values in the vector space $X$ :

$$
\langle x, y\rangle_{s}=(x-(s \pi)(x))(\pi(y))-(y-(s \pi)(y))(\pi(x))
$$

for any $x, y \in Y$.

A subspace $L \subset Y$ is called isotropic for the bilinear form $\langle\cdot, \cdot\rangle_{s}$, if $\left\langle y_{1}, y_{2}\right\rangle_{s}=$ 0 for every pair $\left(y_{1}, y_{2}\right) \in Y \times Y$.

Lemma 2 If $s_{1}: Z \longrightarrow Y$ and $s_{2}: Z \longrightarrow Y$ are two splittings of the exact sequence (2), such that the subspace Image $\left(s_{1}\right)$ is isotropic for the bilinear form $\langle\cdot, \cdot\rangle_{s_{2}}$, then the subspace Image $\left(s_{2}\right)$ is isotropic for the bilinear form $\langle\cdot, \cdot\rangle_{s_{1}}$ and the two bilinear forms $\langle\cdot, \cdot\rangle_{s_{2}}$ and $\langle\cdot, \cdot\rangle_{s_{1}}$, coincide.

Proof. Any splitting $s: Z \longrightarrow Y$ defines an isomorphism

$$
Y \cong Z \times \operatorname{Hom}(Z, X)
$$

and the bilinear form $\langle\cdot, \cdot\rangle_{s}$ on the space $Y$ is the pull-back of the bilinear form $\langle\cdot, \cdot\rangle$ on the space $Z \times \operatorname{Hom}(Z, X)$, which is defined as

$$
\langle(u, \alpha),(v, \beta)\rangle=\alpha(v)-\beta(u)
$$

Any subspace $Z^{\prime} \subset Z \times \operatorname{Hom}(Z, X)$, which is a complement of the subspace $\{0\} \times \operatorname{Hom}(Z, X)$ can be given as

$$
Z^{\prime}=\{(z, f(z)) \mid z \in Z\}
$$

where $f: Z \longrightarrow \operatorname{Hom}(Z, X)$ is a linear mapping.

For any two elements $\left(z_{1}, f\left(z_{1}\right)\right)$ and $\left(z_{2}, f\left(z_{2}\right)\right)$ from the subspace $Z^{\prime}$ we have

$$
\left\langle\left(z_{1}, f\left(z_{1}\right)\right),\left(z_{2}, f\left(z_{2}\right)\right)\right\rangle=f\left(z_{1}\right)\left(z_{2}\right)-f\left(z_{2}\right)\left(z_{1}\right)
$$


Therefore, the subspace $Z^{\prime}$ is isotropic if and only if

$$
f\left(z_{1}\right)\left(z_{2}\right)=f\left(z_{2}\right)\left(z_{1}\right)
$$

for every $z_{1}$ and $z_{2}$ from the space $Z^{\prime}$.

Any element $(z, \alpha) \in Z \times \operatorname{Hom}(Z, X)$ can be represented as

$$
(z, f(z))+(0, \alpha-f(z))
$$

where $(z, f(z)) \in Z^{\prime}$ and $(0, \alpha-f(z)) \in \operatorname{Hom}(Z, X)$. Therefore, the bilinear form defined by the subspace $Z^{\prime}$ is

$$
\begin{gathered}
\left\langle\left(z_{1}, \alpha\right),\left(z_{2}, \beta\right)\right\rangle_{Z^{\prime}}=\left(\alpha-f\left(z_{1}\right)\right)\left(z_{2}\right)-\left(\beta-f\left(z_{2}\right)\right)\left(z_{1}\right)= \\
=\left\langle\left(z_{1}, \alpha\right),\left(z_{2}, \beta\right)\right\rangle-\left(f\left(z_{1}\right)\left(z_{2}\right)-f\left(z_{2}\right)\left(z_{1}\right)\right) .
\end{gathered}
$$

which shows that, the bilinear forms $\langle\cdot, \cdot\rangle_{Z^{\prime}}$ and $\langle\cdot, \cdot\rangle$ are equal if and only if the subspace $Z^{\prime}$ is an isotropic subspace for the bilinear form $\langle\cdot, \cdot\rangle$.

Let us introduce the following fiber bundles over the total space of the Grassmanization $\mathrm{Gr}_{k}(\mathrm{~T}(M))$ :

$\pi_{\Theta}: \Theta \longrightarrow \operatorname{Gr}_{k}(\mathrm{~T}(M))$ be the canonical fiber bundle over the Grassmannization of the tangent bundle $\mathrm{T}(M)$. That is: the fiber of the bundle $\pi_{\Theta}$ at a point $W \in \operatorname{Gr}_{k}\left(\mathrm{~T}_{x}(M)\right)$ is the vector space $W$;

$\pi_{K}: K \longrightarrow \mathrm{Gr}_{k}(\mathrm{~T}(M))$ be the fiber bundle corresponding to the Cartan distribution (see Definition 5) on the manifold $\operatorname{Gr}_{k}(\mathrm{~T}(M))$. That is: the fiber at a point $W \in \operatorname{Gr}_{k}\left(\mathrm{~T}_{x}(M)\right)$ is the Cartan subspace $K_{W}$;

$\pi_{\Upsilon}: \Upsilon \longrightarrow \operatorname{Gr}_{k}(\mathrm{~T}(M))$ be the fiber bundle of the vertical subspaces for the Grassmannian fiber bundle $\widetilde{\tau}_{k}: \operatorname{Gr}_{k}(\mathrm{~T}(M)) \longrightarrow M$. The fiber at a point $W \in \operatorname{Gr}_{k}(\mathrm{~T}(M))$ is the tangent space of the fiber $\operatorname{Gr}_{k}\left(\mathrm{~T}_{x}(M)\right)$, where $W$ is a subspace of the space $\mathrm{T}_{x}(M)$ (as it was mentioned early, this space is isomorphic to the space $\left.\operatorname{Hom}\left(W, \mathrm{~T}_{x}(M) / W\right)\right)$.

We have the following exact sequence of the fiber bundles over the manifold $\operatorname{Gr}_{k}(\mathrm{~T}(M))$

$$
0 \longrightarrow \Theta \stackrel{\imath}{\hookrightarrow} K \stackrel{\left(\widetilde{\tau}_{k}\right)^{\prime}}{\longrightarrow} \Upsilon \longrightarrow 0
$$

where 0 denotes here the trivial fiber bundle with the fibers equal to $\{0\}$.

In other words, for any $x \in M$ and $W \in \mathrm{Gr}_{k}\left(\mathrm{~T}_{x}(M)\right)$, we have the following exact sequence (see 11)

$$
0 \longrightarrow \operatorname{Hom}\left(W, \mathrm{~T}_{x}(M) / W\right) \stackrel{\iota}{\hookrightarrow} K_{W} \stackrel{\left(\widetilde{\tau}_{k}\right)^{\prime}(W)}{\longrightarrow} W \longrightarrow 0
$$

For any fixed $x \in M$ and $W \in \operatorname{Gr}_{k}\left(\mathrm{~T}_{x}(M)\right)$ consider a submanifold $N \subset M$, such that $x \in N$ and $\mathrm{T}_{x}(N)=W$. The submanifold $N$ defines a section (Gauss mapping)

$$
g_{N}:\left.N \longrightarrow \mathrm{Gr}_{k}(\mathrm{~T}(M))\right|_{N}
$$

where by $\left.\operatorname{Gr}_{k}(\mathrm{~T}(M))\right|_{N}$ we denote the restriction of the Grassmann fiber bundle to the submanifold $N$ :

$$
g_{N}(x)=\mathrm{T}_{x}(N) \subset \mathrm{T}_{x}(M)
$$


The Gauss mapping $g_{N}$ induces a mapping $s_{N}: W \longrightarrow K_{W}$ which is a splitting of the exact sequence 1 and is defined as

$$
s_{N}(\xi)=g_{N}^{\prime}(x)(\xi)
$$

for any $\xi \in W=\mathrm{T}_{x}(N)$. On the other side, this splitting defines a bilinear form $\langle\cdot, \cdot\rangle_{N}$ on the vector space $K_{W}$ (see the formula 3), with values in the quotient space $\mathrm{T}_{x}(M) / W$.

Lemma 3 The bilinear form $\langle\cdot, \cdot\rangle_{N}$ is independent of the choice of the submanifold $N$; i.e., if $N^{\prime}$ is another submanifold of the manifold $M$, such that $x \in N^{\prime}$ and $T_{x}\left(N^{\prime}\right)=W$, then the bilinear forms $\langle\cdot, \cdot\rangle_{N^{\prime}}$ and $\langle\cdot, \cdot\rangle_{N}$ are equal.

Proof. As it follows from the Lemma 2, it is sufficient to prove that the space Image $\left(g_{N_{1}}^{\prime}(x)\right)$ is isotropic subspace of the space $K_{W}$, for the bilinear form $\langle\cdot, \cdot\rangle_{N}$.

Using a local linearization of the situation, it is sufficient to consider the case when $N=F$ and $M=F \times E$, where $F$ and $E$ are finite-dimensional real vector spaces, and

$$
N_{1}=\left\{(t, f(t)) \mid t \in F, x=(0,0) \text { and } f(0)=f^{\prime}(0)=0\right\}
$$

In this case we have that the total space of the Grassmannian fiber bundle is

$$
\operatorname{Gr}_{k}(\mathrm{~T}(M))=F \times E \times \operatorname{Gr}_{k}(F \times E)
$$

the subspace $K_{F \times\{0\}}$ of the vector space $\mathrm{T}_{(0,0, F)}\left(F \times E \times \operatorname{Gr}_{k}(F \times E)\right)$, corresponding to the Cartan distribution, is isomorphic to the vector space $F \times$ $\operatorname{Hom}(F, E)$, and the bilinear form on $K_{F \times\{0\}}$, corresponding to the submanifold $F \times\{0\}$ is

$$
\left\langle\left(\xi_{1}, \alpha_{1}\right),\left(\xi_{2}, \alpha_{2}\right)\right\rangle_{F}=\alpha_{1}\left(\xi_{2}\right)-\alpha_{2}\left(\xi_{1}\right)
$$

The Gauss mapping corresponding to the submanifold $N_{1}$ is

$$
g_{N_{1}}(x)=\left\{\left(\xi, f^{\prime}(x)(\xi)\right) \mid \xi \in F\right\}
$$

The corresponding splitting at the point $(0,0) \in N_{1}$

$$
s_{N_{1}}: F \longrightarrow F \times \operatorname{Hom}(F, E)
$$

is defined as

$$
s_{N_{1}}(\xi)=\left(\xi, f^{\prime \prime}(0)(\xi, \cdot)\right)
$$

for any $\xi \in F$. The image of this mapping is isotropic because the bilinear mapping $f^{\prime \prime}(0): F \times F \longrightarrow E$, corresponding to the second derivation is always symmetric.

Let the fiber bundle

$$
\widetilde{\tau}_{k}^{*}(\mathrm{~T}(M)) \longrightarrow \mathrm{Gr}_{k}(\mathrm{~T}(M))
$$


be the pull-back of the tangent bundle of the manifold $M$ to the total space of the Grassmannization of the tangent bundle. Consider the fiber bundle

$$
\tilde{\pi}_{\Theta}: \widetilde{\Theta} \longrightarrow \operatorname{Gr}_{k}(\mathrm{~T}(M))
$$

which is the quotient of the fiber bundle $\widetilde{\tau}_{k}{ }^{*}(\mathrm{~T}(M)) \longrightarrow \mathrm{Gr}_{k}(\mathrm{~T}(M))$ by the canonical fiber bundle $\pi_{\Theta}: \Theta \longrightarrow \operatorname{Gr}_{k}(\mathrm{~T}(M))$ over the total space of the Grassmanization of the tangent bundle; i.e., the fiber of the bundle $\widetilde{\pi}_{\Theta}$ at a point $W \in \operatorname{Gr}_{k}\left(\mathrm{~T}_{x}(M)\right)$ is the quotient space $\mathrm{T}_{x}(M) / W$. Keeping in mind the Lemma 3, we can state that on the fiber bundle which corresponds to the Cartan distribution, there is a canonical bilinear form with values in the fiber bundle $\tilde{\pi}_{\Theta}: \tilde{\Theta} \longrightarrow \mathrm{Gr}_{k}(\mathrm{~T}(M))$

$$
\langle\cdot, \cdot\rangle: K \oplus K \longrightarrow \tilde{\Theta}
$$

Let $D: M \longrightarrow \mathrm{Gr}_{k}(\mathrm{~T}(M))$ be a smooth distribution on the manifold $M$. It is clear that for each point $x \in M$, the mapping

$$
D^{\prime}(x): \mathrm{T}_{x}(M) \longrightarrow \mathrm{T}_{D(x)}\left(\operatorname{Gr}_{k}(\mathrm{~T}(M))\right)
$$

carries the subspace $D(x) \subset \mathrm{T}_{x}(M)$ into the space $K_{D(x)}$ which is the subspace correspondent to the Cartan distribution, at the point $D(x) \in \mathrm{Gr}_{k}(\mathrm{~T}(M))$.

Theorem 1 The distribution $D: M \longrightarrow G r_{k}(T(M))$ is integrable if and only if, for each point $x \in M$, the subspace $D^{\prime}(x)(D(x)) \subset K_{D(x)}$ is isotropic for the bilinear form $\langle\cdot, \cdot\rangle: K_{D(x)} \times K_{D(x)} \longrightarrow T_{x}(M) / D(x)$

Proof. For a given distribution $D: M \longrightarrow \mathrm{Gr}_{k}(\mathrm{~T}(M))$ consider the following bilinear mapping of the fiber bundles

$$
\sigma: D \oplus D \longrightarrow \mathrm{T}(M) / D
$$

where, for each $x \in M$, the mapping

$$
\sigma_{x}: D(x) \times D(x) \longrightarrow \mathrm{T}_{x}(M) / D(x)
$$

is defined as $\sigma_{x}(u, v)=q([\widetilde{u}, \widetilde{v}])$, where $\widetilde{u}$ and $\widetilde{v}$ are vector fields on the manifold $M$, such that $\{\tilde{u}, \tilde{v}\} \subset D, \widetilde{u}(x)=u, \widetilde{v}(x)=v$ and the mapping

$$
q: \mathrm{T}_{x}(M) \longrightarrow \mathrm{T}_{x}(M) / D(x)
$$

is the natural quotient mapping.

The bilinear form $\sigma_{x}$ is defined correctly, i.e., the value $q([\tilde{u}, \tilde{v}])$ is independent of the choice of the extensions $\widetilde{u}$ and $\widetilde{v}$. To check this, consider a vector field $\tau$, on the manifold $M$, such that $\tau \in D$ and $\tau(x)=0$. Let $\left\{D_{1}, \ldots, D_{k}\right\}$ be a local basis of the distribution $D$, and $\tau=\sum_{i} \varphi_{i} D_{i}$, where $\varphi_{i}, i=1, \ldots, k$ are $\mathrm{C}^{\infty}$ class functions. 
For any vector field $\xi \in D$, we have the following

$$
\begin{gathered}
{[\tau, \xi]_{x}=\left[\sum_{i} \phi_{i} D_{i}, \xi\right]_{x}=} \\
=\sum_{i} \underbrace{\phi_{i}(x)}_{0}\left[D_{i}, \xi\right]+\sum_{i} \xi\left(\phi_{i}\right)(x) D_{i}(x) \in D(x) \Rightarrow \\
\Rightarrow \quad q\left([\tau, \xi]_{x}\right)=0
\end{gathered}
$$

Using the linearization, introduced in the proof of Lemma 3, it is easy to see that the pull-back of the bilinear form $\langle\cdot, \cdot\rangle$ by the mapping

$$
D: M \longrightarrow \operatorname{Gr}_{k}(\mathrm{~T}(M))
$$

on the subspaces $D(x) \subset \mathrm{T}_{x}(M)$ coincides with the form $\sigma_{x}$, i.e., for any $u$ and $v \in D(x)$ we have that

$$
\sigma_{x}(u, v)=\left\langle D^{\prime}(x)(u), D^{\prime}(x)(v)\right\rangle
$$

After this, the statement of the theorem is equivalent to the Frobenius classical theorem about the integrability of distributions.

Let $V^{k}(M), k=1, \ldots, \infty$, be the space of antisymmetric, covariant tensor fields on the manifold $M$, and $\Omega^{k}(M), k=1, \ldots, \infty$, be the space of differential $k$-forms on the manifold $M$. Also, we put that $V^{0}(M)=\Omega^{0}(M)=C^{\infty}(M)$.

If $D$ is a submodule of the $C^{\infty}(M)$-module $V^{1}(M)$, then for any point $x \in M$, we have a subspace of the tangent space of the manifold $M$ at the point $x$, generated by the set of vectors $\{\xi(x) \mid \forall \xi \in D\}$ denoted by $D(x)$. Also, any vector field $\xi \in V^{1}(M)$, such that $\xi(x) \in D(x)$ for all $x \in M$, is an element of the submodule $D$. In the case when the dimensions of the subspaces $D(x) \subset \mathrm{T}_{x}(M), x \in M$ are equal to each other, we have the structure referred as distribution, but in some cases the subspaces $D(x) \subset \mathrm{T}_{x}(M), x \in M$ have different dimensions. In this case, the mapping $x \mapsto D(x)$ is referred as a singular distribution.

There is an analogue of the Frobenius theorem for singular distributions (see [25]) which states that the distribution $D$ (singular or regular) is integrable if and only if $D$ is involutive and for any vector field $\xi \in D$, the dimensions of the subspaces $D(x) \subset \mathrm{T}_{x}(M)$ are constant along the integral paths of the vector field $\xi$. 


\section{Derivation Based Noncommutative Differen- tial Calculus}

In noncommutative geometry, the commutative algebra of smooth functions on a smooth manifold is replaced by an abstract algebra, which, in general, can be noncommutative (see, for example [9, 14]). The definitions of the classical geometric objects are translated on the language of the commutative algebra of the smooth functions and than they are generalized to the abstract algebra. In this section, we review the definitions and some facts about diff-geometrical objects on the language of the noncommutative differential geometry.

\subsection{Noncommutative Differential Forms.}

Let $A$ be an associative algebra over the field of real or complex numbers. The space of derivations of the algebra $A$ is the set of such linear mappings

$$
X: A \longrightarrow A
$$

that for each $a, b \in A$ :

$$
X(a b)=X(a) b+a X(b)
$$

It is clear that the space $\operatorname{Der}(A)$ is a Lie algebra and if the algebra $A$ is commutative, then $\operatorname{Der}(A)$ is an $A$-module. Generally, the space $\operatorname{Der}(A)$ is a $Z(A)$ module, where $Z(A)$ denotes the center of the algebra $A$.

There are two noncommutative generalizations of the graded differential algebra of differential forms (see [14], [16]). The first one is $C_{Z(A)}(\operatorname{Der}(A), A)$, which is the graded algebra of antisymmetric $Z(A)$-multilinear mappings from $\operatorname{Der}(A)$ to $A$. We put that

$$
C_{Z(A)}^{0}(\operatorname{Der}(A), A)=A .
$$

The differential operator

$$
\mathrm{d}: C_{Z(A)}^{n}(\operatorname{Der}(A), A) \longrightarrow C_{Z(A)}^{n+1}(\operatorname{Der}(A), A)
$$

is defined by the well-known Koszul formula: for any $\omega \in C_{Z(A)}^{n}(\operatorname{Der}(A), A)$ and $X_{1}, \ldots, X_{n+1} \in \operatorname{Der}(A)$ let

$$
\begin{aligned}
& (\mathrm{d} \omega)\left(X_{1}, \ldots, X_{n+1}\right)= \\
& =\sum_{i=1}^{n+1}(-1)^{i+1} X_{i} \omega\left(X_{1}, \ldots, \hat{X}_{i}, \ldots, X_{n+1}\right)+ \\
& +\sum_{1 \leq i<j \leq n+1}(-1)^{i+j} \omega\left(\left[X_{i}, X_{j}\right], \ldots, \hat{X}_{i}, \ldots, \hat{X}_{j}, \ldots, X_{n+1}\right)
\end{aligned}
$$

We denote the space $C_{Z(A)}(\operatorname{Der}(A), A)$ by $\Omega_{Z}(A)$.

The second generalization of the differential forms over the algebra $A$ is the smallest differential graded subalgebra of the algebra $\Omega_{Z}(A)$ containing the 
algebra $A$. We denote this algebra simply by $\Omega(A)$. Each element $\omega \in \Omega(A)$ can be expressed as a finite sum of the elements of the type $a_{0} \mathrm{~d} a_{1} \cdots \mathrm{d} a_{n}$, where $\mathrm{d} a \in \Omega^{1}(A)$ is the one form defined as:

$$
(\mathrm{d} a)(X)=X(a), \text { for every } X \in \operatorname{Der}(A) .
$$

The multiplication operation in the space $\Omega(A)$ is same as in the space $\Omega_{Z}(A)$.

There is a generalization of the classical operator of the inner derivation $i_{X}: \Omega_{Z}^{n}(A) \longrightarrow \Omega_{Z}^{n+1}(A)$ for any $X \in \operatorname{Der}(A)$, defined as

$$
\begin{aligned}
& \left(i_{X} \omega\right)\left(X_{1}, \ldots, X_{n-1}\right)=\omega\left(X, X_{1}, \ldots, X_{n-1}\right) \text { for } \omega \in \Omega_{Z}^{n}(A) \\
& \text { and } \\
& i_{X}(\alpha)=0 \text { for any } \alpha \in \Omega_{Z}^{0}(A)
\end{aligned}
$$

The subalgebra $\Omega(A)$ is invariant under the action of the operator $i_{X}$.

We shall also use, the noncommutative generalization of the classical Lie derivation operator: $L_{X}: \Omega^{n}(A) \longrightarrow \Omega^{n}(A)$, defined as

$$
L_{X}=i_{X} \circ \mathrm{d}+\mathrm{d} \circ i_{X}
$$

\subsection{Noncommutative Submanifold.}

Let $N$ be a closed submanifold of a smooth compact manifold $M$. We have the following exact sequence of the commutative algebras

$$
0 \longrightarrow I(N) \hookrightarrow C^{\infty}(M) \stackrel{r}{\longrightarrow} C^{\infty}(N) \longrightarrow 0
$$

where $r: C^{\infty}(M) \longrightarrow C^{\infty}(N)$ is the restriction mapping and $I(N)$ is the ideal in the algebra $C^{\infty}(M)$ consisting of functions vanishing on the submanifold $N$.

Let $V_{N}^{1}(M)$ be the subspace of $V^{1}(M)$ consisting of such vector fields $X$ on the manifold $M$, that $X(I(N)) \subset I(N)$. It is clear that if $X \in V_{N}^{1}(M)$, then the restriction of $X$ to the submanifold $N$ is tangent to $N$, and vice versa: any vector field $\xi \in V^{1}(N)$ can be extended to a vector field $X \in V^{1}(M)$, such that $\left.X\right|_{N}=\xi$. Therefore, the restriction mapping

$$
r: V_{N}^{1}(M) \longrightarrow V^{1}(N)
$$

is a surjective mapping. The kernel of this mapping is the set of vector fields on the manifold $M$ vanishing on the submanifold $N$. In other words

$$
(X \in \operatorname{kernel}(r)) \Longleftrightarrow\left(X\left(C^{\infty}(M)\right) \subset I(N)\right) .
$$

Denote the space $\operatorname{kernel}(r)$ by $V_{N}^{1}(M)_{0}$. Hence, we have the following exact sequence of a Lie algebra homomorphisms

$$
0 \longrightarrow V_{N}^{1}(M)_{0} \hookrightarrow V_{N}^{1}(M) \stackrel{r}{\longrightarrow} V^{1}(N) \longrightarrow 0
$$

To translate these structures on the language of the noncommutative geometry, consider an associative real or complex algebra $A$. Let $I$ be an ideal in the 
algebra $A$. Denote by $S_{I}$ the quotient algebra $A / I$ and $q: A \longrightarrow S_{I}$ be the natural quotient mapping.

Consider the following Lie subalgebras in $\operatorname{Der}(A)$ :

$$
\begin{aligned}
& \operatorname{Der}_{I}(A)=\{X \in \operatorname{Der}(A) \mid X(I) \subset I\} \\
& \operatorname{Der}_{I}(A)_{0}=\{X \in \operatorname{Der}(A) \mid X(A) \subset I\}
\end{aligned}
$$

It is clear that the Lie algebra $\operatorname{Der}_{I}(A)_{0}$ is an ideal in the Lie algebra $\operatorname{Der}_{I}(A)$. There is a mapping $r_{I}: \operatorname{Der}_{I}(A) \longrightarrow \operatorname{Der}\left(S_{I}\right)$, defined as

$$
r_{I}(X)(q(a))=q(X(a))
$$

for each $a \in A$ and $X \in \operatorname{Der}_{I}(A)$. This mapping is the noncommutative analogue of the restriction mapping $V_{N}^{1}(M) \longrightarrow V^{1}(N)$, which assigns to a vector field on the manifold $M$, tangent to the submanifold $N$, its restriction to $N$. The kernel of this mapping is exactly the Lie algebra $\operatorname{Der}_{I}(A)_{0}$.

Definition 6 (see [43]) The quotient algebra $S_{I}=A / I$ is called a submanifold algebra of the algebra $A$ if the mapping

$$
r_{I}: \operatorname{Der}_{I}(A) \longrightarrow \operatorname{Der}\left(S_{I}\right)
$$

is surjective. The ideal $I$ in the algebra $A$ is called the constraint ideal for the submanifold.

Hence, if the quotient algebra $S_{I}$ is a submanifold algebra, we have the following exact sequence of Lie algebra homomorphisms

$$
0 \longrightarrow \operatorname{Der}_{I}(A)_{0} \hookrightarrow \operatorname{Der}_{I}(A) \longrightarrow \operatorname{Der}(A / I) \longrightarrow 0
$$

\subsection{Noncommutative Quotient Manifold.}

As before, let $A$ be a real or complex associative algebra and $B$ be its subalgebra. Consider the following Lie subalgebras of the Lie algebra $\operatorname{Der}(A)$ (see 43]):

$$
\begin{aligned}
Q_{B} & =\{X \in \operatorname{Der}(A) \mid X(B) \subset B\} \\
V_{B} & =\{X \in \operatorname{Der}(A) \mid X(B)=0\} .
\end{aligned}
$$

The subalgebra $V_{B}$ is an ideal in the Lie algebra $Q_{B}$, i.e., $\left[V_{B}, Q_{B}\right] \subset V_{B}$. We have a natural restriction mapping

$$
r_{B}: Q_{B} \longrightarrow \operatorname{Der}(B)
$$

which is a Lie algebra homomorphism, and the kernel of this mapping is exactly the Lie algebra $V_{B}$.

Definition 7 (see [43]) The subalgebra $B$ of the algebra $A$ is called a quotient manifold algebra of $A$, if the following conditions are true: 
(q1) $Z(B)=B \cap Z(A)$

(q2) $\operatorname{Der}(B) \cong Q_{B} / V_{B}$

(q3) $B=\left\{a \in A \mid X(a)=0, \forall X \in V_{B}\right\}$

Notice, that the condition (q1) is always true if the algebra $A$ is commutative, and the condition (q2) is equivalent to the restriction mapping $r_{B}: Q_{B} \longrightarrow$ $\operatorname{Der}(B)$ be surjective. In the latter case we have the following short exact sequence

$$
0 \longrightarrow V_{B} \hookrightarrow Q_{B} \longrightarrow \operatorname{Der}(B) \longrightarrow 0
$$

\subsection{Noncommutative Connection and Curvature.}

A bimodule $\Gamma$ over the associative real or complex algebra $A$, is called a central bimodule over the algebra $A$, if $\Gamma$ is also a module over the center of the algebra $A$; i.e., for each $s \in \Gamma$ and each $a \in Z(A)$, where $Z(A)$ is the center of $A$, we have that $a s=s a$.

Let a $Z(A)$-module $L$ be a Lie algebra, and we have a Lie algebra representation of $L$ in the Lie algebra of derivations $\operatorname{Der}(A)$, which is also a $Z(A)$-module homomorphism. Let for any $z \in Z(A)$ and $X, Y \in L$, we have that

$$
[X, z \cdot Y]=X(z) \cdot Y+z \cdot[X, Y]
$$

Definition $8 A$ connection for a pair $(L, \Gamma)$, where $L$ and $\Gamma$ are the same as above, is a mapping

$$
X \mapsto \nabla_{X}
$$

where $X \in L$ and $\nabla_{X}$ is a linear operator

$$
\nabla_{X}: \Gamma \longrightarrow \Gamma
$$

satisfying the following conditions:

(c1) for any $z \in Z(A), a, b \in A$ and $s \in \Gamma$ :

$$
\nabla_{(z \cdot X)}(s)=z \cdot \nabla_{X}(s)
$$

(c2) $\nabla_{X}(a \cdot s \cdot b)=X(a) \cdot s \cdot b+a \cdot\left(\nabla_{X}(s)\right) \cdot b+a \cdot s \cdot X(b)$

The mapping $\nabla: L \longrightarrow \operatorname{Hom}(\Gamma, \Gamma)$, is not necessarily a Lie algebra homomorphism. For any $X, Y \in L$, the mapping

$$
R(X, Y): \Gamma \longrightarrow \Gamma
$$


which measures its deviation from being a homomorphism of Lie algebras is called the curvature of the connection $\nabla$ (see 15]). More explicitly, for any $X, Y \in L$ we have that

$$
R(X, Y)=\left[\nabla_{X}, \nabla_{Y}\right]-\nabla_{[X, Y]}
$$

The mapping $R(X, Y): \Gamma \longrightarrow \Gamma$ is an $A$-bimodule endomorphism, for any $X, Y \in L$.

\subsection{Connection Compatible with Group Action.}

For any subalgebra $B \subset A$, the algebra $A$ is a central bimodule over the algebra $B$. Let $B$ is a quotient manifold algebra in the algebra $A$ and

$$
s: \operatorname{Der}(B) \longrightarrow Q_{B}
$$

be a splitting of the exact sequence 6 , which is a homomorphism of the $Z(B)$ modules, but not necessarily a Lie algebra homomorphism. Such splitting defines a connection for the pair $(\operatorname{Der}(B), A)($ see 43$])$ : for any $X \in \operatorname{Der}(B)$, let the mapping $\nabla_{X}: A \longrightarrow A$ be $\nabla_{X}(a)=s(X)(a)$, for any $a \in A$.

The curvature of this connection is exactly the deviation os the mapping $s$ from being a Lie algebra homomorphism:

$$
R_{\nabla}(X, Y)=[s(X), s(Y)]-s([X, Y]) .
$$

Let $M$ be a smooth manifold and $G$ be a Lie group which acts on the manifold $M$. Let $A$ be the algebra of smooth functions on the manifold $M$ and $B$ be the subalgebra of the algebra $A$, consisting of the functions invariant under the action of the group $G$. If this action is such that the quotient space $M / G$ is a smooth manifold, then the subalgebra $B$ is a quotient manifold algebra of $A$; i.e., for any vector field $X$ on the manifold $M / G$, exists a vector field $Y$ on the manifold $M$, such that $q^{\prime}(Y)=X$, where $q: M \longrightarrow M / G$ is the quotient mapping. As the mapping $q$ is surjective, the mapping

$$
q^{\prime}(x): \mathrm{T}_{x}(M) \longrightarrow \mathrm{T}_{q(x)}(M / G)
$$

is also surjective for any point $x \in M$. If the quotient space $M / G$ is a manifold, the vector field $Y$ is a section of the subbundle of the tangent bundle $\mathrm{T}(M)$, the fiber of which at a point $x \in M$ is the space $q^{\prime}(x)^{-1}(X(q(x)))$.

Let $g$ be the Lie algebra of the Lie group $G$, and the mapping

$$
o: g \longrightarrow \operatorname{Der}(A)
$$

be the natural Lie algebra homomorphism induced by the action of the group $G$ on the manifold $M$; i.e., for any $x \in M$ and $u \in g$, let

$$
o(u)=\phi^{\prime}(1, x)(u, 0),
$$

where $\phi: G \times M \longrightarrow M$ is the mapping defining the group action. The Lie algebra $V_{B}$ is the submodule in the $A$-module $\operatorname{Der}(A)$ generated by the subspace Image $(o)$, and the subalgebra $Q_{B}$ is the maximal subspace of $\operatorname{Der}(A)$ such that $\left[\right.$ Image $\left.(o), Q_{B}\right] \subset V_{B}$ (or, equivalently: $\left.\left[V_{B}, Q_{B}\right] \subset V_{B}\right)$. 
Definition 9 A connection compatible with the action of the Lie group $G$, is a splitting $s: \operatorname{Der}(B) \longrightarrow Q_{B}$ of $Z(B)$-modules, such that for any $X \in \operatorname{Der}(B)$ and $u \in g$, we have that $[o(u), s(x)]=0$.

Now, let us translate this construction on the language of the noncommutative geometry.

The group action on a manifold can be generalized as a Lie subalgebra $g \subset \operatorname{Der}(A)$. The submodule of the vertical vector fields corresponding to this action can be described as the $A$-submodule $V^{g} \subset \operatorname{Der}(A)$ generated by $g$. Let $B^{g}$ be the subalgebra of the algebra $A$, such that, for any $b \in B^{g}$ and $v \in V^{g}: v(b)=0$. Let $Q^{g}$ be the maximal subspace of $\operatorname{Der}(A)$ such that $\left[V^{g}, Q^{g}\right] \subset V^{g}$. It is clear that $Q^{g}$ is a Lie subalgebra of the Lie algebra $\operatorname{Der}(A)$ and is a module over $Z\left(B^{g}\right)$. Recall that $V_{B^{g}}$ is such subspace of $\operatorname{Der}(A)$ that $V_{B^{g}}\left(B^{g}\right)=\{0\}$, and $Q_{B^{g}}$ is such that $Q_{B^{g}}\left(B^{g}\right) \subset B$.

Lemma 4 The space $Q^{g}$ is a subspace of the space $Q_{B^{g}}$.

Proof. For any $\xi \in Q^{g}, b \in B^{g}$ and $v \in V^{g}$ we have the following:

$$
v(\xi(b))=\underbrace{[v, \xi]}_{\in V}(b)-\xi(\underbrace{v(b)}_{0})=0,
$$

therefore: $\xi(b) \in B$.

It is clear that $V^{g} \subset V_{B^{g}}$ but in general, these two Lie algebras are not identical.

Proposition 1 If $V^{g}=V_{B^{g}}$ then $Q^{g}=Q_{B^{g}}$.

Proof. We have already proved that $Q^{g}$ is always a subspace of $Q_{B^{g}}$. Now the task is to prove that if $V^{g}=V_{B^{g}}$ then $Q_{B^{g}}$ is a subspace of the space $Q^{g}$. It is equivalent to $\left[V^{g}, Q_{B^{g}}\right] \subset V^{g}$. But if $V^{g}=V_{B^{g}}$ this statement is the same as $V^{g}$ be a Lie ideal in the Lie algebra $Q_{B^{g}}$, which follows from the fact that $V^{g}$ is a kernel of the Lie algebra homomorphism

$$
r_{B^{g}}: Q_{B^{g}} \longrightarrow \operatorname{Der}\left(B_{g}\right)
$$

(see the short exact sequence 6).

Definition 10 In the case when $V^{g}=V_{B^{g}}$, and the subalgebra $B^{g}$ in the algebra $A$ is a quotient manifold subalgebra, a connection compatible with the Lie subalgebra $g \subset \operatorname{Der}(A)$ is a splitting

$$
s: \operatorname{Der}\left(B^{g}\right) \longrightarrow Q^{g}
$$

such that $[g, s(x)]=0$ for any $x \in \operatorname{Der}\left(B^{g}\right)$. 


\subsection{Noncommutative Distribution. Integral Manifold and Bott Connection.}

Further, any submodule of the $Z(A)$-module $\operatorname{Der}(A)$, where $A$ is an associative complex or real algebra, we call a distribution. If a distribution $D$ is a Lie subalgebra of the Lie algebra $\operatorname{Der}(A)$, it will be said to be an involutive distribution.

Definition 11 Let I be an ideal in the algebra A, such that the quotient algebra $S_{I}=A / I$ is a submanifold algebra (see Definition Q). The quotient algebra $S_{I}$ is said to be a integral submanifold algebra for the distribution $D$, if $D$ is is a subalgebra of the Lie algebra $\operatorname{Der}_{I}(A)$ and $r_{I}(D)=\operatorname{Der}\left(S_{I}\right)$, where $r_{I}: \operatorname{Der}_{I}(A) \longrightarrow \operatorname{Der}\left(S_{I}\right)$ is defined by the formula S.

In other words, if the "submanifold " $A / I$ is integral for the distribution $D$, then for each $X \in D$, we have that $X(I) \subset I$; and any "vector field" $Y \in \operatorname{Der}(A / I)$ can be extended to the "vector field" $X \in D$.

For a given distribution $D$, let us denote by $A_{D}$ the subalgebra of the algebra $A$ defined as

$$
A_{D}=\{a \in A \mid X(a)=0, \forall X \in D\}
$$

In the classical geometric situation, if $D$ is ivolutive and regular distribution on some smooth manifold $M$, the algebra $A_{D}$ coincides with the subalgebra of the smooth functions on $M$, constants along the leaves of the foliation, defined by the distribution $D$. In some "good" cases, when the quotient space of the manifold $M$, by the integral submanifolds of the distribution $D$ is a smooth manifold, the subalgebra $A_{D}$ is, in fact, the algebra of smooth functions on this quotient manifold. In such situations, the algebra $A_{I}$ is a quotient manifold algebra in the sense of the noncommutative definition (see Definition 7 ).

Let $L$ be a Lie algebra and a module over a commutative algebra $A$, satisfying the condition 7 . Let $L_{0}$ be a Lie subalgebra of the Lie algebra $L$, and le $L_{0}$ is also a submodule of the $A$-module $L$. In this situation, the quotient space $\Gamma=L / L_{0}$ inherits an $A$-module structure from $L$ and there is a canonical connection for the pair $\left(L_{0}, \Gamma=L / L_{0}\right)$, defined as

$$
\nabla_{X}(q(u))=q([X, u])
$$

for any $X \in L_{0}$ and $u \in L$, where $q$ is the natural quotient mapping, assigning to each $u \in L$, its equivalency class $q(u) \in L / L_{0}$. This definition is correct, i.e., for any two elements $u_{1}, u_{2} \in L$, if $q\left(u_{1}\right)=q\left(u_{2}\right)$ then $\nabla_{X}\left(q\left(u_{1}\right)\right)=\nabla_{X}\left(q\left(u_{2}\right)\right)$. To check this, recall that by definition

$$
\nabla_{X}\left(q\left(u_{1}\right)\right)-\nabla_{X}\left(q\left(u_{2}\right)\right)=q\left(\left[X, u_{1}-u_{2}\right]\right),
$$

and the latter is equal to 0 , because: $X, u_{1}-u_{2} \in L_{0}$ and $L_{0}$ is a Lie subalgebra of $L$.

The mapping $X \mapsto \nabla_{X}$ satisfies the conditions (c1), (c2) required for a connection (see Definition 8): 
for any $a \in A$, we have

$$
\begin{aligned}
& \nabla_{a X}(q(u))=q([a X, u])=q(\underbrace{u(a) X}_{\in L_{0}}+a[X, u])= \\
& =q(a[X, u])=a q([X, u])=a \nabla_{X}(q(u))
\end{aligned}
$$

and

$$
\begin{aligned}
& \nabla_{X}(a q(u))=\nabla_{X}(q(a u))=q([X, a u])= \\
& =q(X(a) u)+q(a[X, u])=X(a) q(u)+a \nabla_{X}(q(u))
\end{aligned}
$$

Now, let us give some kind of dual definition of the connection described above.

Denote by $\Omega^{1}(L, A)$ the space of $A$-valued 1 -forms on the $A$-module $L$, i.e., each element $\alpha \in \Omega^{1}(L, A)$ is a mapping $\alpha: L \longrightarrow A$, such that for any $X \in L$ and $a \in A$ we have that $\alpha(a X)=a \alpha(X)$.

For any Lie subalgebra $L_{0} \in L$, which is also a submodule, let us denote by $\Omega^{1}\left(L, A, L_{0}\right)$ the submodule of the $A$-module $\Omega^{1}(L, A)$ consisting of the forms vanishing on the submodule $L_{0}$.

Define a connection $\nabla$, for the pair $\left(L_{0}, \Omega^{1}\left(L, A, L_{0}\right)\right)$ as follows: for any $X \in L_{0}$ and $\alpha \in \Omega^{1}\left(L, A, L_{0}\right)$, let

$$
\nabla_{X}(\alpha)=L_{X}(\alpha)
$$

where $L_{X}: \Omega^{1}(L, A) \longrightarrow \Omega^{1}(L, A)$ is the operator of Lie derivation:

$$
L_{X}=\mathrm{d} \circ i_{X}+i_{X} \circ \mathrm{d} .
$$

By definition of the space $\Omega^{1}\left(L, A, L_{0}\right)$, we have that for any $\alpha \in \Omega^{1}\left(L, A, L_{0}\right)$ and $X \in L_{0}: i_{X}(\alpha)=0$. Therefore, the formula 9 can be simplified as

$$
\nabla_{X}(\alpha)=\left(i_{X} \circ \mathrm{d}\right)(\alpha)
$$

Lemma 5 For any $X \in L_{0}$, the subspace $\Omega^{1}\left(L, A, L_{0}\right)$ in the space $\Omega^{1}(L, A)$ is invariant under the action of the operator $\nabla_{X}$.

Proof. For $\alpha \in \Omega^{1}\left(L, A, L_{0}\right)$ and $X, Y \in L_{0}$, we have

$$
\begin{aligned}
& \left(\nabla_{X}(\alpha)\right)(Y)=\left(\left(i_{X} \circ \mathrm{d}\right)(\alpha)\right)(Y)=(\mathrm{d} \alpha)(X, Y)= \\
& =X \alpha(Y)-Y \alpha(X)-\alpha([X, Y])=0 .
\end{aligned}
$$


Lemma 6 The connection $\nabla$ for the pair $\left(L_{0}, \Omega^{1}\left(L, A, L_{0}\right)\right)$ is flat

Proof. For any $X, Y \in L_{0}$, we have

$$
R(X, Y)=\left[\nabla_{X}, \nabla_{Y}\right]-\nabla_{[X, Y]}=\left[L_{X}, L_{Y}\right]-L_{[X, Y]}=0
$$

which follows from the well-known fact that the mapping $X \mapsto L_{X}$ is a Lie algebra homomorphism.

To apply the construction described above to the classical geometric case, consider the case when $L$ is the algebra of smooth vector fields on a smooth manifold $M$ and $L_{0}=D \subset V^{1}(M)$ be any involutive distribution on the manifold $M$. If the distribution $D$ is regular, then as it follows from the Frobenius theorem, it is integrable. The natural connection for the pair $\left(D, \Omega_{D}^{1}(M)\right)$, where $\Omega_{D}^{1}(M)$ denotes the space of 1 -forms vanishing on the vector fields from $D$, is in fact, the well-known Bott connection for the foliation of the integral leaves of the distribution $D$. More precisely:

let $N \subset M$ be an integral submanifold of the distribution $D$. Consider a fiber bundle

$$
\pi: E_{D} \longrightarrow N
$$

the fiber of which at a point $x \in N$ is the quotient space $\mathrm{T}_{x}(M) / \mathrm{T}_{x}(N)$ (or, in the dual terminology: the subspace of the space $\mathrm{T}_{x}^{*}(M)$ consisting of the 1-forms vanishing on the subspace $\left.\mathrm{T}_{x}(M)\right)$. The connection for the pair $\left(D, \Omega_{D}^{1}(M)\right)$ corresponds to some ordinary connection on the fiber bundle $\pi: E_{D} \longrightarrow N$, which is known as the Bott connection on a leave of a foliation. A strict algebraic definition of this connection in the terms of noncommutative submanifolds will be given further.

Let $I \subset A$ be an ideal, invariant under the action of the Lie subalgebra $L_{0} \subset L$. That is, for any $X \in L_{0}$ and $a \in I$, we have $X(a) \in L$. Introduce the following two spaces:

$$
\begin{aligned}
& I \cdot L_{0}=\left\{k_{1} u_{1}+\cdots+k_{n} u_{n} \mid k_{1}, \ldots, k_{n} \in I, u_{1}, \ldots, u_{n} \in L\right\} \\
& \text { and } \\
& I \cdot \Omega^{1}\left(L, A, L_{0}\right)= \\
& =\left\{k_{1} \alpha_{1}+\cdots+k_{n} \alpha_{n} \mid k_{1}, \ldots, k_{n} \in I, \alpha_{1}, \ldots, \alpha_{n} \in \Omega^{1}\left(L, A, L_{0}\right)\right\}
\end{aligned}
$$

Lemma 7 For any $X \in L_{0}$, the submodule $I \cdot \Omega^{1}\left(L, A, L_{0}\right)$ is invariant under the action of the covariant derivation $\nabla_{X}$

Proof. For any $X \in L_{0}, a \in I$ and $\alpha \in \Omega^{1}\left(L, A, L_{0}\right)$ we have the following

$$
\begin{aligned}
& \nabla_{X}(a \alpha)=i_{X}(\mathrm{~d}(a \alpha))= \\
& =i_{X}((\mathrm{~d} a) \alpha+a(\mathrm{~d} \alpha))=X(a) \alpha+a \nabla_{X}(\alpha) .
\end{aligned}
$$


As the ideal $I$ is invariant under the action of the Lie subalgebra $L_{0}$, we have that $X(a) \in I$; a submodule $\Omega^{1}\left(L, A, L_{0}\right)$ is invariant under the Lie derivation, we have that $\nabla_{X}(\alpha) \in \Omega^{1}\left(L, A, L_{0}\right)$. Consequently, we obtain that $\nabla_{X}(a \alpha) \in$ $I \cdot \Omega^{1}\left(L, A, L_{0}\right)$.

It is easy to check that the submodule $I \cdot L_{0}$ is a Lie ideal in the Lie algebra $L_{0}$, that is, for any $a \in I, u, v \in L_{0}:[a u, v] \in L_{0}$. As $I \cdot \Omega^{1}\left(L, A, L_{0}\right)$ is invariant under the action of the Lie algebra $L_{0}$, we can define the action of $L_{0}$ on the quotient module

$$
\Omega^{1}\left(L, A, L_{0}\right) / I \cdot \Omega^{1}\left(L, A, L_{0}\right) .
$$

Lemma 8 The action of the Lie subalgebra $I \cdot L_{0} \subset L_{0}$ on the quotient module $\Omega^{1}\left(L, A, L_{0}\right) / I \cdot \Omega^{1}\left(L, A, L_{0}\right)$ is trivial.

Proof. It is equivalent to the statement that any element of the Lie algebra $I \cdot L_{0}$ carries the elements of the space $\Omega^{1}\left(L, A, L_{0}\right)$ in the subspace $I \cdot \Omega^{1}\left(L, A, L_{0}\right)$.

Consider $a \in I, u \in L_{0}$ and $\alpha \in \Omega^{1}\left(L, A, L_{0}\right)$. For these elements, we have the following

$$
\nabla_{(a u)}(\alpha)=i_{(a u)}(\mathrm{d} \alpha)=a i_{u}(\mathrm{~d} \alpha)=a \nabla_{u}(\alpha) \in I \cdot \Omega^{1}\left(L, A, L_{0}\right) .
$$

As the submodule $I \cdot L_{0}$ is a Lie algebra ideal in $L_{0}$, we have that the quotient module $L_{0} / I \cdot L_{0}$ is a Lie algebra and it follows from the Lemma 7 and 8 , that the natural connection on the pair $\left(L_{0}, \Omega^{1}\left(L, A, L_{0}\right)\right)$ induces a connection on the pair

$$
\left(L_{0} / I \cdot L_{0}, \Omega^{1}\left(L, A, L_{0}\right) / I \cdot \Omega^{1}\left(L, A, L_{0}\right)\right)
$$

In the case of the classical geometry, the subalgebra $L_{0}$ corresponds to an involutive distribution $D$ on a smooth manifold $M ; I$ is an ideal of smooth functions on the manifold $M$, corresponding to some integral submanifold of the distribution $D$. The quotient Lie algebra $L_{0} / I \cdot L_{0}$ can be identified with the Lie algebra of the vector fields on the submanifold $N$; and the quotient module $\Omega^{1}\left(L, A, L_{0}\right) / I \cdot \Omega^{1}\left(L, A, L_{0}\right)$ - to the module of the sections of the fiber bundle $\pi: E_{D} \longrightarrow N$.

Now, we describe another algebraic model for the Bott connection on an integral submanifold.

Let $D \subset \operatorname{Der}(A)$ be a distribution (i.e., a Lie subalgebra) and $I$ be an ideal in the algebra $A$, such that the quotient algebra $A / I$ is an integral submanifold algebra for the distribution $D$. As it follows from the definition of the noncommutative integral submanifold (see Definition 11), we have the following short exact sequence

$$
0 \longrightarrow D_{I} \hookrightarrow D \stackrel{r_{I}}{\longrightarrow} \operatorname{Der}(A / I) \longrightarrow 0
$$

where $D_{I}$ is the subalgebra of the Lie algebra $D$, that can be described as

$$
D_{I}=\{X \in D \mid X(A) \subset I\}
$$


Let $\Omega_{D}^{1}(A)$ be the submodule of the module $\Omega^{1}(A)$ consisting of the 1 -forms vanishing on the vector fields from the distribution $D$. As it follows from our previous discussion, there is a natural connection for the pair $\left(D, \Omega_{D}^{1}(A)\right)$. Let us give an algebraic description of the Bott connection on an integral submanifold of a distribution.

Let $\Omega^{1}(A, I)$ be the submodule of $\Omega^{1}(A)$, consisting of such forms $\alpha$, that for any $X \in \operatorname{Der}(A): \alpha(X) \in I$.

Remark 1 In the sense of the classical geometry, the space $\Omega^{1}(A, I)$ corresponds to the submodule of the forms vanishing on the submanifold constrained by the ideal $I$.

Denote by $\Gamma(D, I)$ the quotient module $\Omega_{D}^{1}(A) /\left(\Omega_{D}^{1}(A) \cap \Omega^{1}(A, I)\right)$.

For each $U \in D / D_{I} \cong \operatorname{Der}(A / I)$ define an operator

$$
\nabla_{U}: \Gamma(D, I) \longrightarrow \Gamma(D, I)
$$

as

$$
\nabla_{U}\left(q_{2}(\alpha)\right)=q_{2}\left(L_{X}(\alpha)\right)
$$

where $u=q_{1}(x)$ and the mappings

$$
\begin{aligned}
& q_{1}: D \longrightarrow D / D_{I} \\
& q_{2}: \Omega_{D}^{1}(A) \longrightarrow \Omega_{D}^{1}(A) /\left(\Omega_{D}^{1}(A) \cap \Omega^{1}(A, I)\right)
\end{aligned}
$$

are the canonical projection mappings.

Proposition 2 The correspondence $U \mapsto \nabla_{U}$ is a connection for the pair $(\operatorname{Der}(A / I), \Gamma(D, I))$.

Proof. It is sufficient to check that the definition of the operator $\nabla_{U}$ is correct. For this, we should check that for any $V \in D_{I}$ and $\alpha \in \Omega_{D}^{1}(A): L_{V}(\alpha) \in$ $\Omega^{1}(A, I)$. By definition, we have that for any element $X \in \operatorname{Der}(A)$

$$
\begin{aligned}
& \left(L_{V}(\alpha)\right)(X)=\left(\left(i_{V} \circ \mathrm{d}\right)(\alpha)\right)(X)= \\
& =V \alpha(X)-X \alpha(V)-\alpha([V, X])
\end{aligned}
$$

From the definition of the Lie ideal $D_{I}$ follows that $V \alpha(X) \in I$.

From the definition of $\Omega_{D}^{1}(A)$, follows that $\alpha(V)=0$.

To show that $\alpha([V, X]) \in I$, recall that the form $\alpha$ can be represented as $\alpha=$ $\sum_{i} a_{i} \mathrm{~d} b_{i}$ where $a_{i}, b_{i} \in A$. Therefore

$$
\begin{aligned}
\alpha([V, X]) & =V\left(\sum_{i} a_{i} X\left(b_{i}\right)\right)-X \alpha(V)= \\
& =V\left(\sum_{i} a_{i} X\left(b_{i}\right)\right) \in I
\end{aligned}
$$

which follows from the fact that $\alpha(V)=0$ and $V \in D_{I}$.

Definition 12 The connection for the pair $(\operatorname{Der}(A / I), \Gamma(D, I))$ is called the Bott connection for the integral manifold constrained by the ideal I. 


\section{Lie Superalgebra Structures on the Space of Multiderivations}

\subsection{Compositional Product and Supercommutator}

Let $V$ be a vector space over the field of real or complex numbers. For each integer $k \geq 0$ let us denote by $L^{k}(V)$ the space of multilinear antisymmetric mappings from $V^{k}$ into $V$. We also set that

$$
L^{0}(V)=V \text { and } L(V)=\bigoplus_{k=0}^{\infty} L^{k}(V)
$$

There is a natural associative algebra structure on the space $L^{1}(V)$, defined by the composition of the elements as linear operators, and a natural Lie algebra structure induced by the above associative algebra structure, where the Lie bracket is the ordinary commutator of two linear operators:

$$
[\alpha, \beta]=\alpha \circ \beta-\beta \circ \alpha
$$

for all $\alpha, \beta \in L^{1}(V)$.

The multiplication operation (composition) on the space $L^{1}(V)$ can be extended to the space $L(V)$ and the resulted operator is called the compositional product (see [44): for $\alpha \in L^{m}(V)$ and $\beta \in L^{n}(V)$ the compositional product $\alpha \circ \beta \in L^{m+n-1}(V)$ is

$$
\begin{aligned}
& (\alpha \circ \beta)\left(a_{1}, \ldots, a_{m+n-1}\right)= \\
& =\sum_{s} \operatorname{sgn}(s) \alpha(\beta(\underbrace{a_{s(1)}, \ldots, a_{s(n)}}_{s(1)<\cdots<s(n)}), \underbrace{a_{s(n+1)}, \ldots, a_{s(m+n-1)}}_{s(n+1)<\cdots<s(m+n-1)})
\end{aligned}
$$

where $a_{1}, \ldots, a_{m+n-1}$ are elements of the vector space $V$ and $\operatorname{sgn}(s)$ is the signature of the permutation $s$.

As a result, the commutator existing on the space $L^{1}(V)$ can be extended to the operation on the space $L(V)$, called a supercommutator:

$$
[\alpha, \beta]=(-1)^{(m+1) n} \alpha \circ \beta+(-1)^{m} \beta \circ \alpha
$$

This operation (bracket) satisfies the following conditions, which makes the space $L(a)$ a Lie superalgebra: for $\alpha \in L^{m}(V), \beta \in L^{n}(V)$ and $\gamma \in L^{k}(V)$

(s1) $[\alpha, \beta]=(-1)^{m n}[\beta, \alpha]$;

(s2) $(-1)^{m k}[[\alpha, \beta], \gamma]+(-1)^{m n}[[\beta, \gamma], \alpha]+(-1)^{n k}[[\gamma, \alpha], \beta]=0$

An element $\mu \in L^{2}(V)$ satisfying the condition $[\mu, \mu]=0$, we call an involutive element. Such element defines a Lie algebra structure on the vector space $V$, 
via the commutator

$$
[a, b]_{\mu}=\mu(a, b)
$$

for $a, b \in V$. Notice, that the Jacoby identity for the commutator $[\cdot, \cdot]_{\mu}$ is equivalent to the condition $[\mu, \mu]=0$. Any involutive element $\mu \in L^{2}(V)$ defines a linear opeator

$$
\partial_{\mu}: L(V) \longrightarrow L(V)
$$

as

$$
\partial_{\mu}(\alpha)=[\mu, \alpha]
$$

for any $\alpha \in L(V)$. The degree of this operator is equal to +1 , that is

$$
\partial_{\mu}\left(L^{k}(V)\right) \subset L^{k+1}(V)
$$

for any integer $k \geq 0$.

From the condition (s2) for the commutator $[\cdot, \cdot]$ and the fact that the element $\mu \in L^{2}(V)$ is involutive, easily follows that the operator $\partial_{\mu}$ is a coboundary operator: $\partial_{\mu} \circ \partial_{\mu}=0$.

\subsection{Supercommutator on the Space of Multiderivations}

Now, let $V=A$ be a real or complex commutative algebra. In this case, the space is endowed with a structure of exterior algebra under the multiplication operation defined by the classical formula. For $\alpha \in L^{m}(A)$ and $\beta \in L^{n}(A)$ we have that

$$
\begin{aligned}
& (\alpha \wedge \beta)\left(u_{1}, \ldots, u_{m+n}\right)= \\
& =\frac{1}{m ! n !} \sum_{s} \operatorname{sgn}(s) \alpha\left(u_{s(1)}, \ldots, u_{s(m)}\right) \beta\left(u_{s(m+1)}, \ldots, u_{s(m+n)}\right)
\end{aligned}
$$

where $\left\{u_{1}, \ldots, u_{m+n}\right\} \subset A$.

Definition 13 an element $\alpha \in L(A)$ is said to be a multiderivation if for any set of elements $\left\{a, a_{1}, \ldots, a_{k}\right\} \subset A$ we have that

$$
\alpha\left(a a_{1}, a_{2}, \ldots, a_{k}\right)=a \alpha\left(a_{1}, \ldots, a_{k}\right)+a_{1} \alpha\left(a, a_{2}, \ldots, a_{k}\right)
$$

For any integer $k \geq 0$ the subspace of all multiderivations in the space $L^{k}(A)$ we denote by $\operatorname{Der}^{k}(A)$. Also, we set that $\operatorname{Der}^{0}(A)=A$ and $\operatorname{Der}(A)=\bigoplus_{k}^{\infty} \operatorname{Der}^{k}(A)$.

The subspace $\operatorname{Der}(A)$ in the space $L(A)$ is closed as under the operation of the exterior multiplication defined by the formula 14, so under the supercommutator defined by the formula 13. Moreover, these two structures are interconnected via the following property

$$
[\alpha, \beta \gamma]=[\alpha, \beta] \wedge \gamma+(-1)^{(m+1) n} \beta \wedge[\alpha, \gamma]
$$

for any $\alpha \in \operatorname{Der}^{m}(A), \beta \in \operatorname{Der}^{n}(A)$ and $\gamma \in \operatorname{Der}(A)$. 
In the classical case, when $A=C^{\infty}(M)$, the supercommutator $[\cdot, \cdot]$ on the space $\operatorname{Der}(A)$ is called the Schouten bracket.

For any integer $k \geq 0$ consider $\wedge^{k} \operatorname{Der}^{1}(A)$ which is the subspace of the space $\operatorname{Der}^{k}(A)$, consisting of the linear combinations of the elements of the type $v_{1} \wedge \cdots \wedge v_{k},\left\{v_{1}, \ldots, v_{k}\right\} \subset \operatorname{Der}^{1}(A)$. The space

$$
\wedge \operatorname{Der}^{1}(A)=\bigoplus_{k=0}^{\infty} \wedge^{k} \operatorname{Der}^{1}(A)
$$

is a subalgebra in the exterior algebra $\operatorname{Der}(A)$ and also is closed under the supercommutator. The explicit formula for the restriction of the supercommutator on the subspace $\wedge \operatorname{Der}^{1}(A)$ is the following:

$$
\begin{aligned}
& {\left[u_{1} \wedge \ldots \wedge u_{m}, v_{1} \wedge \ldots \wedge v_{n}\right]=} \\
& =\sum_{i, j}(-1)^{m+i+j-1}\left[u_{i}, v_{j}\right] \wedge u_{1} \wedge \cdots \wedge \hat{u}_{i} \wedge \cdots \wedge u_{m} \wedge \\
& \wedge v_{1} \wedge \cdots \wedge \hat{v}_{j} \wedge \cdots \wedge v_{n}
\end{aligned}
$$

where $u_{1}, \ldots, u_{m}$ and $v_{1}, \ldots, v_{n}$ are the elements of the space $\operatorname{Der}^{1}(A)$.

\subsection{Poisson Structure. Poisson Cohomologies}

As it was mentioned early, an involutive element $P \in L^{2}(A)$ defines a commutator $[\cdot, \cdot]_{P}: A \times A \longrightarrow A$. In the case when $P \in \operatorname{Der}^{2}(A)$, this commutator is a biderivation. That is: for any $a, b$ and $c \in A$, we have

$$
[a, b \cdot c]=b \cdot[a, c]+[a, b] \cdot c
$$

Definition $14 A$ Poisson algebra is a real or complex vector space A, with two operations

$$
(a, b) \mapsto a b \quad \text { and } \quad(a, b) \mapsto\{a, b\}
$$

satisfying the following three conditions:

(p1) the operation (multiplication) $(a, b) \mapsto a b$ makes $A$ an associative algebra;

(p2) the operation (bracket) $(a, b) \mapsto\{a, b\}$ makes $A$ a Lie algebra;

(p3) these two operations are related via the Leibnitz rule:

$$
\{a, b \cdot c\}=b \cdot\{a, c\}+\{a, b\} \cdot c
$$


So, it can be stated that, an involutive element $P \in \operatorname{Der}^{2}(A)$ defines a Poisson algebra structure on the commutative algebra $A$ :

$$
\{a, b\}_{P}=P(a, b)
$$

As the subspace $\operatorname{Der}(A) \subset L(A)$ is closed under the supercommutator, it will be invariant under the action of the coboundary operator $\partial_{P}$. Therefore, we have the subcomplex $\left(\operatorname{Der}(A), \partial_{P}\right)$ of the complex $\left(L(A), \partial_{P}\right)$.

From the condition 15, for the commutator $[\cdot, \cdot]$ on the exterior algebra $\operatorname{Der}(A)$, follows that the operator $\partial_{P}: \operatorname{Der}(A) \longrightarrow \operatorname{Der}(A)$ is an antidifferential, that is: for any $u \in \operatorname{Der}^{m}(A)$ and $v \in \operatorname{Der}^{n}(A)$

$$
\partial_{P}(u \wedge v)=\partial_{P}(u) \wedge v+(-1)^{m} u \wedge \partial_{P}(v)
$$

Therefore, the exterior algebra structure on the space $\operatorname{Der}(A)$, induces an exterior algebra structure on the cohomologies of the complex $\left(\operatorname{Der}(A), \partial_{P}\right)$.

The cohomology algebra of the complex $\left(\operatorname{Der}(A), \partial_{P}\right)$, is said to be the cohmologies of the Poisson structure $(A, P)$ or simply, the Poisson cohomologies.

\subsection{External Differential as a Supercommutator}

For a real or complex vector space $V$, let us denote by $\operatorname{End}(V)$ the space of linear endomorphisms of the space $V$. If $V$ is a module over the commutative algebra $A$, we denote by $\operatorname{End}_{A}(V)$ the space of $A$ - modular endomorphisms of the space $V$. It is clear that $\operatorname{End}_{A}(V) \subset \operatorname{End}(V)$. For any element $a \in A$, the operator of the multiplication on the element $a(v \mapsto a \cdot v)$, we denote by $\widehat{a}$.

An element $\phi \in \operatorname{End}(V)$ is said to be a first order differential operator on the $A$-module $V$, if for any $a \in A$ we have that $[\phi, \widehat{a}] \in \operatorname{End}_{A}(V)$. The space of first order differential operators on the $A$-module $V$ we denote by $\operatorname{Diff}^{1}(V)$ (see 55 ). The space $\operatorname{Diff}^{1}(V)$ is an $A$-module.

It is clear that for the commutative algebra $A$, the mapping $a \mapsto \widehat{a}$ gives an isomorphism $A \cong \operatorname{End}_{A}(A)$.

Lemma 9 The module Diff ${ }^{1}(V)$ is canonically isomorphic to the module $\operatorname{Der}^{1}(A) \oplus$ A.

Proof. Any element $\phi \in \operatorname{Diff}^{1}(A)$ can be represented as

$$
\phi=(\phi-\widehat{\phi(1)})+\widehat{\phi(1)}
$$

As it follows from the definition of a first order differential operator, for any $a \in A$, the operator $[\phi, \widehat{a}]$ is an element of the space $\operatorname{End}_{A}(A)$. Therefore, for any $b \in A$, we have

$$
[\phi, \widehat{a}](b)=b[\phi, \widehat{a}](1)
$$

expanding the last equality, we obtain

$$
\phi(a b)+a b \phi(1)=a \phi(b)+\phi(a) b
$$


subtracting from the last equality the term $2 \cdot \phi(1) a b$, we easily obtain the equality

$$
(\phi-\widehat{\phi(1)})(a b)=a \cdot(\phi-\widehat{\phi(1)})(b)+(\phi-\widehat{\phi(1)})(a) \cdot b
$$

which means that the operator $\phi-\widehat{\phi(1)}: A \longrightarrow A$ is a derivation.

The superalgebra structure defined at the beginning of this section, on the space $L(V)$, where $V$ is a real or complex vector space can be considered in the case when $V=\operatorname{Diff}^{1}(A)$. The space $\operatorname{Diff}^{1}(A)$ is equipped with the natural structure of a Lie algebra defined by the commutator: $[u, v]=u \circ v-v \circ$ $u$, for $u, v \in \operatorname{Diff}^{1}(A)$, which means that, there exists an involutive element $\mu \in L^{2}\left(\operatorname{Diff}^{1}(A)\right)$, such that $[u, v]=\mu(u, v)$. The element $\mu$, itself, defines the coboundary operator

$$
\partial_{\mu}=[\mu, \cdot]: L\left(\operatorname{Diff}^{1}(A)\right) \longrightarrow L\left(\operatorname{Diff}^{1}(A)\right)
$$

So, we can talk about the differential complex $\left(L\left(\operatorname{Diff}^{1}(A)\right), \partial_{\mu}\right)$.

For each integer $n \geq 1$, let $\widetilde{L}^{n}\left(\operatorname{Diff}^{1}(A)\right)$ be the subspace of the space $L^{n}\left(\operatorname{Diff}^{1}(A)\right)$, consisting of the mappings

$$
\omega: \underbrace{\operatorname{Diff}^{1}(A) \times \cdots \times \operatorname{Diff}^{1}(A)}_{n-\text { times }} \rightarrow \operatorname{Diff}^{1}(A)
$$

satisfying the following conditions:

- the values of the mapping $\omega$ are in $A$ (recall that the space $\operatorname{Diff}^{1}(A)$ is isomorphic to (see Lemma 9) $\operatorname{Der}^{1}(A) \oplus A$ );

- $\omega\left(u_{1}, \ldots, u_{n}\right)=0$ if at least one of the elements $\left\{u_{1}, \ldots, u_{n}\right\} \subset \operatorname{Diff}^{1}(A)$ is in $A$;

- $\omega$ is $A$-multilinear mapping; i.e.

$$
\omega\left(a \cdot u_{1}, \ldots, u_{n}\right)=a \cdot \omega\left(u_{1}, \ldots, u_{n}\right)
$$

for any $a \in A$ and any collection $\left\{u_{1}, \ldots, u_{n}\right\} \subset \operatorname{Diff}^{1}(A)$.

Also, we set that $\widetilde{L}^{0}\left(\operatorname{Diff}^{1}(A)\right)=A$.

It is easy to see that the space $\widetilde{L}^{n}\left(\operatorname{Diff}^{1}(A)\right)$ is the same as the space of derivation based differential forms for the commutative algebra $A: C_{Z(A)}^{n}(\operatorname{Der}(A), A)$ (see Section 2.1).

Proposition 3 The subspace $\widetilde{L}\left(\operatorname{Diff}^{1}(A)\right)=\bigoplus_{n=0}^{\infty} \widetilde{L}^{n}\left(\operatorname{Diff}^{1}(A)\right)$ in the space $L\left(\operatorname{Diff}^{1}(A)\right)$ is invariant under the action of the operator $\partial \mu=[\mu, \cdot]$ and the restriction of the operator $\partial \mu$ on the subalgebra $\widetilde{L}\left(D_{i f f}{ }^{1}(A)\right)$, coincides with the classical Koszul differential on the space of differential forms (see Section 2.1). 
Proof. By definition of the compositional product and the corresponding supercommutator (see Formula 13 and Formula 12) we have the following

$$
\begin{aligned}
& {[\mu, \omega]\left(u_{1}, \ldots, u_{n+1}\right)=} \\
& =(-1)^{n} \sum(-1)^{n+1-i} \mu\left(\omega\left(u_{1}, \ldots, \widehat{u}_{i}, \ldots, u_{n+1}\right), u_{i}\right)+ \\
& +\sum(-1)^{i+j-1} \omega\left(\mu\left(u_{i}, u_{j}\right), u_{1}, \ldots, \widehat{u}_{i}, \ldots, \widehat{u}_{j}, \ldots, u_{n+1}\right)= \\
& =\sum(-1)^{i-1}\left[\omega\left(u_{1}, \ldots, \widehat{u}_{i}, \ldots, u_{n+1}\right), u_{i}\right]+ \\
& +\sum(-1)^{i+j-1} \omega\left(\left[u_{i}, u_{j}\right], u_{1}, \ldots, \widehat{u}_{i}, \ldots, \widehat{u}_{j}, \ldots, u_{n+1}\right)= \\
& =\sum(-1)^{i} u_{i} \omega\left(u_{1}, \ldots, \widehat{u}_{i}, \ldots, u_{n+1}\right)+ \\
& +\sum(-1)^{i+j-1} \omega\left(\left[u_{i}, u_{j}\right], u_{1}, \ldots, \widehat{u}_{i}, \ldots, \widehat{u}_{j}, \ldots, u_{n+1}\right)= \\
& =-\sum(-1)^{i-1} u_{i} \omega\left(u_{1}, \ldots, \widehat{u}_{i}, \ldots, u_{n+1}\right)+ \\
& +\sum(-1)^{i+j} \omega\left(\left[u_{i}, u_{j}\right], u_{1}, \ldots, \widehat{u}_{i}, \ldots, \widehat{u}_{j}, \ldots, u_{n+1}\right)= \\
& =-(d \omega)\left(\left(u_{1}, \ldots, u_{n+1}\right)\right)
\end{aligned}
$$

To summarize, we can state that generally, the subspace $\widetilde{L}\left(\operatorname{Diff}^{1}(A)\right)$ in the space $L\left(\operatorname{Diff}^{1}(A)\right)$ is not closed under the operation of supercommutator, but it is invariant under the action of the operator $[\mu, \cdot]$, where $\mu \in L^{2}\left(\operatorname{Diff}^{1}(A)\right)$ is the element, corresponding to the Lie algebra commutator in the space $\operatorname{Diff}^{1}(A)$. It can be defined an operation of external multiplication in the space $\widetilde{L}\left(\operatorname{Diff}^{1}(A)\right) \cong$ $C_{Z(A)}^{n}(\operatorname{Der}(A), A)$ by the formula 14, after which, the operator $\partial \mu$ becomes the antiderivation of degree +1 on the algebra $\widetilde{L}\left(\operatorname{Diff}^{1}(A)\right)$.

For simplicity, further we denote the space $\widetilde{L}\left(\operatorname{Diff}^{1}(A)\right)$ by $\widehat{\Omega}(A)$.

Any element $p \in \operatorname{Der}^{2}(A)$ defines the mapping

$$
\widetilde{p}: A \longrightarrow \operatorname{Der}^{1}(A)
$$

as follows

$$
\widetilde{p}(a)(x)=p(a, x)
$$

which can be extended to the mapping

$$
\widetilde{p}: \widehat{\Omega}(A) \longrightarrow \operatorname{Der}(A)
$$

by the following formula

$$
\widetilde{p}(\alpha)\left(a_{1}, \ldots, a_{n}\right)=(-1)^{n} \alpha\left(\tilde{p}\left(a_{1}\right), \ldots, \tilde{p}\left(a_{n}\right)\right)
$$

where $\alpha \in \widehat{\Omega}^{n}(A)$ and $\left\{a_{1}, \ldots, a_{n}\right\} \subset A$. 
As it was mentioned, the bracket

$$
\{\cdot, \cdot\}: A \times A \longrightarrow A, \quad\{a, b\}=p(a, b)
$$

is a Lie algebra structure on $A$ if and only if the element $p$ is involutive $([p, p]=$ 0 ), because of the following equality

$$
\frac{1}{2}[p, p](a, b, c)=\{\{a, b\}, c\}+\{\{b, c\}, a\}+\{\{c, a\}, b\}
$$

Furthermore, there is true the following

Lemma 10 If the element $p \in \operatorname{Der}^{2}(A)$ is involutive, the mapping

$$
\widetilde{p}: A \longrightarrow \operatorname{Der}^{1}(A)
$$

is a Lie algebra homomorphism.

Proof. The proof easily follows from the following sequence of equalities

$$
\begin{aligned}
& \widetilde{p}(\{a, b\})(c)=\{\{a, b\}, c\}=\{a,\{b, c\}\}-\{b,\{a, c\}\}= \\
& =(\widetilde{p}(a) \widetilde{p}(b)-\widetilde{p}(b) \widetilde{p}(c))(c)
\end{aligned}
$$

The following theorem extends the above homomorphism to higher order elements

Theorem 2 The mapping $\widetilde{p}: \widehat{\Omega}(A) \longrightarrow \operatorname{Der}(A)$ is a homomorphism of the differential complexes $(\widehat{\Omega}(A), d)$ and $\left(\operatorname{Der}(A), \partial_{p}=[p, \cdot]\right)$.

Proof.

$$
\begin{aligned}
& \widetilde{p}(d \omega)\left(a_{1}, \ldots, a_{n+1}\right)= \\
& =(-1)^{n+1}(d \omega)\left(\widetilde{p}\left(a_{1}\right), \ldots, \widetilde{p}\left(a_{n+1}\right)\right)= \\
& =(-1)^{n+1}\left(\sum_{i}(-1)^{i-1} \widetilde{p}\left(a_{i}\right) \omega\left(\widetilde{p}\left(a_{1}\right), \ldots, \widetilde{p}\left(a_{1}\right), \ldots, \widetilde{p}\left(a_{n+1}\right)\right)+\right. \\
& \left.+\sum_{i<j}(-1)^{i+j} \omega\left(\left[\widetilde{p}\left(a_{i}\right), \widetilde{p}\left(a_{j}\right)\right], \ldots, \widehat{p}\left(a_{i}\right), \ldots, \widehat{p}\left(a_{j}\right), \ldots, \widetilde{p}\left(a_{n+1}\right)\right)\right)= \\
& =(-1)^{n+1}\left(\sum _ { i } ( - 1 ) ^ { i - 1 } p \left(a_{i}, \omega\left(\widetilde{p}\left(a_{1}\right), \ldots, \widetilde{p}\left(a_{1}\right), \ldots, \widetilde{p}\left(a_{n+1}\right)\right)+\right.\right. \\
& \left.+\sum_{i<j}(-1)^{i+j} \omega\left(\left[\widetilde{p}\left(a_{i}\right), \widetilde{p}\left(a_{j}\right)\right], \ldots, \widehat{\widetilde{p}\left(a_{i}\right)}, \ldots, \widehat{\widetilde{p}\left(a_{j}\right)}, \ldots, \widetilde{p}\left(a_{n+1}\right)\right)\right)
\end{aligned}
$$


On the other hand we have:

$$
\begin{aligned}
& {[p, \widetilde{p}(\omega)]\left(a_{1}, \ldots, a_{n+1}\right)=} \\
& =\sum_{i}(-1)^{i-1} p\left((\widetilde{p}(\omega))\left(a_{1}, \ldots, \widehat{a_{i}}, \ldots, a_{n+1}\right), a_{i}\right)+ \\
& +\sum_{i<j}(-1)^{i+j-1} \widetilde{p}(\omega)\left(p\left(a_{i}, a_{j}\right), \ldots, \widehat{a_{i}}, \ldots, \widehat{a_{j}}, \ldots, \widetilde{p}\left(a_{n+1}\right)\right)= \\
& =(-1)^{n+1}\left(\sum_{i}(-1)^{i-1} p\left(a_{i}, \omega\left(\widetilde{p}\left(a_{1}\right), \ldots, \widehat{p}\left(a_{i}\right), \ldots, \widetilde{p}\left(a_{n+1}\right)\right)\right)+\right. \\
& \left.+\sum_{i<j}(-1)^{i+j-1} \omega\left(\left[\widetilde{p}\left(a_{i}\right), \widetilde{p}\left(a_{j}\right)\right], \ldots, \widehat{p}\left(\widehat{a_{i}}\right), \ldots, \widetilde{p}\left(\widehat{a_{j}}\right), \ldots, \widetilde{p}\left(a_{n+1}\right)\right)\right)
\end{aligned}
$$




\section{Schouten Bracket as the Deviation of the Coboundary Operator from the Leibnitz Rule}

\subsection{An Invariant Formula for Schouten Bracket}

The main result of the previous section is the fact that a supercommutator and a second order involutive element on an external algebra, defines some coboundary operator on this algebra; and even the classical Koszul differential on the external algebra of differential forms can be represented as a supercommutator with some second order element of some superalgebra containing the algebra of differential forms. In this section, we consider some reversed situation: a coboundary operator on some external algebra induces a supercommutator on this algebra.

Let $E=\bigoplus_{n=0}^{\infty} E_{n}$ be a real or complex $\mathbf{Z}$-graded external algebra with a multiplication operator denoted by $\wedge$. That is: for $\alpha \in E_{m}$ and $\beta \in E_{m}$, we have that $\alpha \wedge \beta \in E_{m+n}$ and $\alpha \wedge \beta=(-1)^{m+n} \beta \alpha$, where $\{m, n\} \subset \mathbf{N}$.

Let $\partial: E \longrightarrow E$ be a boundary operator; i.e., for any $k=1, \ldots, \infty$ :

$$
\partial\left(E_{k}\right) \subset E_{k-1} \text { and } \partial \circ \partial=0 .
$$

The operator $\partial$ is said to be an antidifferential if for any $u \in E_{m}$ and $v \in E_{n}$, satisfies the following condition

$$
\partial(u \wedge v)=\partial(u) \wedge v+(-1)^{m} u \wedge \partial(v)
$$

For any boundary operator $\partial$ on the external algebra $E$ can be defined the bilinear mapping $[\cdot, \cdot]: E \times E \longrightarrow E$ as

$$
[u, v]=\partial(u) \wedge v+(-1)^{m} u \wedge \partial(v)-\partial(u \wedge v)
$$

If the operator $\partial$ is an antidifferential, then the commutator defined by this formula is trivial one: $[u, v]=0$ for any $u, v \in E$.

In any case, the question: is the commutator $[\cdot, \cdot]$ a Lie superalgebra structure on the graded algebra $E$ or not? is natural. To be so, recall that the following conditions must be hold: for any $u \in E_{m}, v \in E_{n}$ and $w \in E_{k}$

(sa1) $[u, v]=(-1)^{m n}[v, u]$

(sa2) $[u, v \wedge w]=[u, v] \wedge w+(-1)^{(m+1) n} v \wedge[u, w]$

(sa3) $(-1)^{m k}[[u, v], w]+(-1)^{m n}[[v, w], u]+(-1)^{n k}[[w, u], v]=0$

The first of these three conditions is obviously always true. The condition (sa3) is also always true for the the first order elements and it can be easily seen that is equivalent to the equality

$$
(\partial \circ \partial)(x \wedge y \wedge z)=0
$$


for any $\{x, y, z\} \subset E_{1}$. This condition is equivalent to the bracket $[x, y]=$ $-\partial(x, y)$ be a Lie algebra structure on the space $E_{1}$.

The condition (sa2) is equivalent to the following equality for the operator $\partial$ :

$$
\begin{aligned}
& \partial(\alpha \wedge \beta \wedge \gamma)= \\
& =\partial(\alpha \wedge \beta) \wedge \gamma+(-1)^{m} \alpha \wedge \partial(\beta \wedge \gamma)+(-1)^{(m+1) n} \beta \wedge \partial(\alpha \wedge \gamma)- \\
& -\left(\partial \alpha \wedge \beta \wedge \gamma+(-1)^{m} \alpha \wedge \partial \beta \wedge \gamma+(-1)^{m+n} \alpha \wedge \beta \wedge \partial \gamma\right)
\end{aligned}
$$

It is easy to check that the condition (sa2) implies that the operator $\partial$, on the elements of the type $u_{1} \wedge, \ldots, \wedge u_{n} \in E_{n}$ where $u_{1}, \ldots, u_{n}$ are elements of the space $E_{1}$, has the following form:

$$
\begin{aligned}
& \partial\left(u_{1} \wedge, \ldots, \wedge u_{n}\right)= \\
& =\sum_{i<j}(-1)^{i+j}\left[u_{i}, u_{j}\right] \wedge u_{1} \wedge \cdots \wedge \widehat{u_{i}} \wedge \cdots \wedge \widehat{u_{j}} \wedge \cdots \wedge u_{n}
\end{aligned}
$$

and in this case, all of the above three conditions are true on the subalgebra $\wedge E_{1}=\sum_{k=0}^{\infty}\left(\wedge^{k} E_{1}\right)$.

Let $L$ is a Lie algebra and a module over the commutative algebra $A$, which itself is a module over the Lie algebra $L$. That is: there is a Lie algebra homomorphism from the Lie algebra $L$ to the Lie algebra of derivations of the algebra $A$, and these two structures are related via the following condition: for any $x, y \in L$ and $a \in A$, we have $[x, a y]=x(a) \cdot y+a \cdot[x, y]$.

For any integer $n>0$, we denote by $\operatorname{Alt}^{n}(L, A)$ the space of all skewsymmetric, multilinear (over the field of real or complex numbers) mappings from $L^{n}$ to $A$. Using the formula 18 for the supercommutator on the space $\wedge L=$ $\sum \wedge^{k} L$, we obtain that for any $u \in \wedge^{m} L, v \in \wedge^{n} L$ and $\omega \in \operatorname{Alt}^{m+n-1}(L, A)$ the following equality is true

$$
\omega([u, v])=\omega(\partial(u) \wedge v)+(-1)^{m} \omega(u \wedge \partial(v))-\omega(\partial(u \wedge v))
$$

or in other notations

$$
\begin{aligned}
& \omega([u, v])= \\
& =(-1)^{(m+1) n} i_{v} \omega(\partial(u))+(-1)^{m} i_{u} \omega(\partial(v))-\omega(\partial(u \wedge v))
\end{aligned}
$$

where, for $\alpha \in \operatorname{Alt}^{p}(L, A)$ and $x \in \wedge^{q} L$, under the expression $i_{x} \alpha$, is denoted the element of the space $\operatorname{Alt}^{p-q}(L, A)$ defined as

$$
\left(i_{x} \alpha\right)(y)=\alpha(x \wedge y)
$$

for any $y \in \wedge^{p-q} L$. 
form

Using the dual notations, the expression 20 can be rewritten in the following

$$
\begin{aligned}
& \omega([u, v])= \\
& =(-1)^{(m+1) n}\left(\partial^{*} i_{v} \omega\right)(u)+(-1)^{m}\left(\partial^{*} i_{u} \omega\right)(v)-\left(\partial^{*} \omega\right)(u \wedge v)
\end{aligned}
$$

where: $\left(\partial^{*} \alpha\right)(x)=\alpha(\partial(x))$, for any $\alpha \in \operatorname{Alt}^{p}(L, A)$ and $x \in \wedge^{p} L$.

By the definition of the classical external differential, we have that $d \alpha=$ $\partial^{*} \alpha+\partial_{1} \alpha$, where, for $u_{1}, \ldots, u_{p+1} \in L$, the expression $\partial_{1} \alpha$ is defined as

$$
\left(\partial_{1} \alpha\right)\left(u_{1} \wedge \ldots \wedge u_{p+1}\right)=\sum_{i=1}^{p+1}(-1)^{i-1} u_{i} \alpha\left(u_{1} \wedge \ldots \wedge \widehat{u_{i}} \wedge \ldots \wedge u_{p+1}\right)
$$

It is easy to verify, that

$$
(-1)^{(m+1) n}\left(\partial_{1} i_{v} \omega\right)(u)+(-1)^{m}\left(\partial_{1} i_{u} \omega\right)(v)-\left(\partial_{1} \omega\right)(u \wedge v)=0
$$

Therefore, in the expression 21, we can replace the operator $\partial^{*}$ by the operator $d$, after which we obtain the following formula for the Schouten bracket:

$$
\begin{aligned}
& \omega([u, v])= \\
& =(-1)^{(|u|+1)|v|}\left(d i_{v} \omega\right)(u)+(-1)^{|u|}\left(d i_{u} \omega\right)(v)-(d \omega)(u \wedge v)
\end{aligned}
$$

where $|u|$ denotes the order of the element $u \in \operatorname{Alt}(L, A)$; i.e., if $u$ is an element of the space $\operatorname{Alt}^{n}(L, A)$, then $|u|=n$. This formula can be considered as an invariant definition of the Schouten bracket in some cases, for example, in the case of covariant, skew-symmetric tensor fields on a smooth manifold.

\subsection{Star Operator for a Poisson Structure. Poisson Coho- mologies.}

As it was mentioned several times, an involutive element $p \in L \wedge L$, defines the operator of degree +1

$$
\partial_{p}=[p, \cdot]: \wedge L \longrightarrow \wedge L
$$

which is a coboundary operator. The dual operator

$$
\partial_{p}^{*}: \operatorname{Alt}(L, A) \longrightarrow \operatorname{Alt}(L, A)
$$

defined as

$$
\left.\partial_{p}^{*} \omega\right)(x)=\omega([p, x]
$$

is a boundary operator $\left(\partial_{p}^{*} \circ \partial_{p}^{*}=0\right)$ of degree -1 . Using the formula 22 we obtain the following expression for $\partial_{p}^{*}$ :

$$
\left(\partial_{p}^{*} \omega\right)(u)=(d \omega)(p \wedge u)-\left(d i_{p} \omega\right)(u)-(-1)^{|u|}\left(d i_{u} \omega\right)(p)
$$


or, in more brief notations

$$
\left(\partial_{p}^{*} \omega\right)(u)=\left(i_{p} \circ d-d \circ i_{p}\right)(\omega)(u)-(-1)^{|u|}\left(d i_{u} \omega\right)(p)
$$

For any integer $n>1$, let $\operatorname{Alt}_{A}^{n}(L, A)$ be the subspace of the space $\operatorname{Alt}^{n}(A)$, consisting of the $A$-multilinear mappings.

It is clear that the subalgebra $\operatorname{Alt}_{A}(L, A)=\sum_{n=0}^{\infty} \operatorname{Alt}_{A}^{n}(L, A)$ of the algebra $\operatorname{Alt}(L, A)$ is not invariant under the action of the operator $\partial_{p}^{*}$, because, for $\omega \in \operatorname{Alt}_{A}^{n}(L, A), a \in A$, and $x \in \wedge^{n} L$, we have

$$
\begin{aligned}
& \left(\partial_{p}^{*} \omega\right)(a \cdot x)=-\omega([p, a \cdot x])=-\omega(\widetilde{p}(a) \wedge x+a[p, x])= \\
& =-\left((-1)^{|x|}\left(i_{x} \omega\right)(\widetilde{p}(a))+a \cdot \omega([p, x])\right)= \\
& =a \cdot\left(\partial_{p}^{*} \omega\right)(x)-(-1)^{|x|}\left(i_{x} \omega\right)(\widetilde{p}(a))
\end{aligned}
$$

To "correct" the operator $\partial_{p}^{*}$, so that the algebra of differential forms $\operatorname{Alt}_{A}(L, A)$ be invariant under its action, we remove the last term in the 23. The result is exactly the boundary operator of the canonical complex for Poisson manifold, which is well-known in the case when $L$ is the Lie algebra of vector fields on some Poisson manifold $M$, and $A$ is the commutative algebra of smooth functions on $M$ (see [5]):

$$
\delta: \operatorname{Alt}_{A}^{m}(L, A) \longrightarrow \operatorname{Alt}_{A}^{m-1}(L, A), \quad \delta=i_{p} \circ \mathrm{d}-\mathrm{d} \circ i_{p}
$$

Consider the following bilinear mapping

$$
\widehat{p}: \operatorname{Alt}_{A}^{m}(L, A) \times \operatorname{Alt}_{A}^{n}(L, A) \longrightarrow \operatorname{Alt}_{A}^{m+n-2}(L, A)
$$

defined as

$$
\widehat{p}(\alpha, \beta)=i_{p}(\alpha \wedge \beta)-i_{p} \alpha \wedge \beta-\alpha \wedge i_{p} \beta
$$

The Schouten bracket on the anticommutative graded algebra

$$
\operatorname{Alt}_{A}(L, A)=\sum_{k=0}^{\infty} \operatorname{Alt}_{A}^{k}(L, A)
$$

can be defined as

$$
[\alpha, \beta]=\mathrm{d} p(\alpha, \beta)-p(\mathrm{~d} \alpha, \beta)-(-1)^{|\alpha|} p(\alpha, \mathrm{d} \beta)
$$

(see 34$)$.

Proposition 4 The bracket on $A_{A}(L, A)$ defined by the formula 26 coincides with the bracket [ . , . ] $\delta$ which is the obstruction for the operator $\delta$ to be an antiderivative. That is: for any $\alpha \in \operatorname{Alt}_{A}^{m}(L, A)$, and $\beta \in A_{A}(L, A)$, the following equality is true

$$
\delta \alpha \wedge \beta+(-1)^{m} \alpha \wedge \beta-\delta(\alpha \wedge \beta)=d p(\alpha, \beta)-p(d \alpha, \beta)-(-1)^{m} p(\alpha, d \beta)
$$


The proof of this proposition consists of a simple verifying of the equation keeping in mind the formulas 24, 25 and 26.

For any $a \in A$, we the 1 -form $\mathrm{d} a \in \operatorname{Alt}_{A}^{1}(L, A)$, as

$$
(\mathrm{d} a)(X)=X(a)
$$

for any $X \in L$. Consider the subalgebra of the algebra $\operatorname{Alt}_{A}(L, A)$ generated by $A$ and $\mathrm{d} A \subset \operatorname{Alt}_{A}^{1}(L, A)$. For simplicity, further, we shall identify this subspace with the entire space $\operatorname{Alt}_{A}(L, A)$. As it follows from the definition, this space consists of the elements of the type

$$
\sum_{i=1}^{n} a_{0}^{i} \mathrm{~d} a_{1}^{i} \wedge \mathrm{d} a_{2}^{i}
$$

The Poisson bracket on $A$ defined by the involutive element $p$, as $\{a, b\}=$ $i_{p}(\mathrm{~d} a \wedge \mathrm{d} b)$, for $a, b \in A$, gives the same expression for the operator $\delta$, as in the case when $A$ is the algebra of smooth functions on some Poisson manifold and $L$ is the Lie algebra of the vector fields on the same manifold (see [月]):

$$
\begin{aligned}
& \delta\left(a_{0} \mathrm{~d} a_{1} \wedge \ldots \wedge a_{n}\right)= \\
& =\sum_{i=1}^{n}(-1)^{i+1}\left\{a_{0}, a_{i}\right\} \mathrm{d} a_{1} \wedge \widehat{\mathrm{d} a_{i}} \wedge \mathrm{d} a_{n}+ \\
& +\sum_{i<j}(-1)^{i+j} a_{0} \mathrm{~d}\left\{a_{i}, a_{j}\right\} \wedge \mathrm{d} a_{1} \wedge \ldots \wedge \widehat{\mathrm{d}}_{i} \wedge \ldots \wedge \widehat{\mathrm{d} a_{j}} \wedge \ldots \wedge \mathrm{d} a_{n}
\end{aligned}
$$

By using of this formula, it is easy to verify that on $\operatorname{Alt}_{A}(L, A)$ the condition 26 for $\delta$ is true. Therefore, the bracket defined by 24 or by

$$
[\alpha, \beta]=\delta \alpha \wedge \beta+(-1)^{|\alpha|} \alpha \wedge \delta \beta-\delta(\alpha \wedge \beta)
$$

on $\operatorname{Alt}_{A}(L, A)$ gives a Lie superalgebra structure, which is the extension of the Lie algebra structure on the space $\operatorname{Alt}_{A}^{1}(L, A)$. So, in the case when $A=C^{\infty}(M)$ for some Poisson manifold $M$, and $L$ is the Lie algebra of vector fields on the same manifold, we can state that the supercommutator of differential forms on $M$ is the obstruction for the canonical boundary operator $\delta$ to be an antidifferential.

An element $\xi=x \wedge y \in L \wedge L$ defines the mapping from $A$ into $L, a \mapsto \widetilde{\xi}(a)$, as

$$
\widetilde{\xi}(a)=x(a) \cdot y-y(a) \cdot x
$$

It is clear that for each $a \in A$, the expression $\widetilde{\xi}(a)$ depends only on $\mathrm{d} a \in$ $\operatorname{Alt}_{A}^{1}(L, A)$. This mapping can be extended linearly for any $p \in L \wedge L$. After that, for any $p \in L \wedge L$ we can define the mappings

$$
\widetilde{p}: \operatorname{Alt}_{A}^{k}(L, A) \longrightarrow \wedge^{k} L, \quad k=0, \ldots, \infty
$$


as follows

$$
\widetilde{p}\left(a_{0} \mathrm{~d} a_{1} \wedge \cdots \wedge \mathrm{d} a_{k}\right)=a_{0} \widetilde{p}\left(a_{1}\right) \wedge \cdots \wedge \widetilde{p}\left(a_{k}\right)
$$

For any fixed $\omega \in \operatorname{Alt}_{A}^{n}(L, A)$, define a series of mappings:

$$
\star: \operatorname{Alt}_{A}^{k}(L, A) \longrightarrow \operatorname{Alt}_{A}^{n-k}(L, A)
$$

as

$$
\star\left(a_{0} \mathrm{~d} a_{1} \wedge \ldots \wedge \mathrm{d} a_{k}\right)=a_{0}\left(i_{\widetilde{p}\left(a_{k}\right)} \circ \cdots \circ i_{\widetilde{p}\left(a_{1}\right)}\right)(\omega)
$$

In the case when $M$ is a symplectic manifold with a symplectic form $\alpha$, the commutative algebra $A$ is $C^{\infty}(M)$, the second order element $p$ is the bivector field corresponding to the form $\alpha$, the Lie algebra $L$ is the Lie algebra of vector fields on $M$, and $\omega=\alpha^{(\operatorname{dim} M) / 2}$, the operator $\star$ is the well-known analogue of the star operator on a Riemannian manifold (see [30], [56], [5]).

Proposition 5 If $\omega$ satisfies the following conditions $d \omega=0$ and $d a \wedge \omega=0$ for each $a \in A$ then the equality $\star \circ \delta=(-1)^{k} d \circ \star$ is true on the algebra $A l t_{A}^{k}(L, A)$, if and only if $\left(d \circ i_{\widetilde{p}(a)}\right) \omega=0$, for any $a \in A$.

Proof. On the space $\operatorname{Alt}_{A}^{1}(L, A)$ we have the following equalities:

$$
(\star \circ \delta)\left(a_{0} \mathrm{~d} a_{1}\right)=\star\left(\left\{a_{0}, a_{1}\right\}\right)=\left\{a_{0}, a_{1}\right\} \cdot \omega
$$

and

Consequently:

$$
(\mathrm{d} \circ \star)\left(a_{0} \mathrm{~d} a_{1}\right)=\mathrm{d}\left(a_{0} i_{\widetilde{p}\left(a_{1}\right)} \omega\right)=\mathrm{d} a_{0} \wedge i_{\widetilde{p}\left(a_{1}\right)} \omega+a_{0} \mathrm{~d} i_{\widetilde{p}\left(a_{1}\right)} \omega
$$

$$
\begin{aligned}
& (\star \circ \delta+\mathrm{d} \circ \star)\left(a_{0} \mathrm{~d} a_{1}\right)= \\
& =\left\{a_{0}, a_{1}\right\} \cdot \omega+\mathrm{d} a_{0} \wedge i_{\widetilde{p}\left(a_{1}\right)} \omega+a_{0} \mathrm{~d} i_{\widetilde{p}\left(a_{1}\right)} \omega= \\
& =-i_{\widetilde{p}\left(a_{1}\right)}\left(\mathrm{d} a_{0} \wedge \omega\right)+a_{0} \mathrm{~d} i_{\widetilde{p}\left(a_{1}\right)} \omega=a_{0} \mathrm{~d} i_{\widetilde{p}\left(a_{1}\right)} \omega
\end{aligned}
$$

Therefore, on the space $\operatorname{Alt}_{A}^{1}(L, A)$ the equality $\star \circ \delta=-\mathrm{d} \circ \star$ is true if and only if $\left(d \circ i_{\widetilde{p}(a)}\right) \omega=0$ for any $a \in A$.

To proof the equality

$$
\star \circ \delta=(-1)^{k} \mathrm{~d} \circ \star
$$

for every space $\operatorname{Alt}_{A}^{k}(L, A), k=0, \ldots, \infty$, the following well-known formula can be used

$$
\begin{aligned}
& \left(L_{X} \omega\right)\left(X_{1}, \ldots, X_{n}\right)= \\
& =\left(i_{X} \mathrm{~d} \omega+\mathrm{d} i_{X} \omega\right)\left(X_{1}, \ldots, X_{n}\right)= \\
& =X \omega\left(X_{1}, \ldots, X_{n}\right)-\sum_{i} \omega\left(X_{1}, \ldots,\left[X, X_{i}\right], \ldots, X_{n}\right)
\end{aligned}
$$

So, we can state that the operator $\star$ induces a homomorphism from the homology space $H_{i}(L, A, \delta)$ of the complex $\left(\operatorname{Alt}_{A}(L, A), \delta\right)$ into the cohomology space $H^{n-i}(L, A)$ of the complex $\left(\operatorname{Alt}_{A}(L, A), \mathrm{d}\right)$. 


\section{Differential Complex and Generalized Func- tions on Poisson Manifold}

\subsection{Brief Overview of Geometric structures on Poisson Manifold}

Further we shall consider the case when the commutative algebra $A$ is the algebra $C^{\infty}(M)$ for some $\mathrm{C}^{\infty}$ class manifold $M$; the Lie algebra $L$ is the Lie algebra of vector fields on the manifold $M$ and therefore $\operatorname{Alt}_{A}(L, A)$ is the exterior algebra of differential forms on $M$, denoted here by $\Omega(M)$. An involutive element $p \in V^{2}(M)$, where $V^{2}(M)$ is the space of the second-order covariant antisymmetric tensor fields on $M$, defining a Poisson algebra structure on the space $C^{\infty}(M)$ is called as a bivector field on the manifold $M$, correspondent to the Poisson structure. For the Poisson bracket of a pair of smooth functions on $M$, we use the common notation: $\{f, g\}$.

In the case when the bivector field $p$ is a non-degenerated covariant tensor field, it defines a second order differential form $\omega$ on the manifold $M$, which is non-degenerated and the condition $[p, p]=0$ is equivalent to $\mathrm{d} \omega=0$. In this case, the differential form $\omega$ is called the symplectic form and the pair $(M, \omega)$ is said to be a symplectic manifold. A vector field $X \in V^{1}(M)$ is said to be symplectic, if $L_{X}(\omega)=0$. For a symplectic manifold $(M, \omega)$, let us denote by $V^{1}(M)_{S}$ the space of all symplectic vector fields on this manifold. From the following well-known equalty $L_{[X, Y]}=\left[L_{X}, L_{Y}\right]$, easily follows that the space $V^{1}(M)_{S}$ is a Lie subalgebra in $V^{1}(M)$, though it is not a $\mathrm{C}^{\infty}(M)$-submodule.

In the case of a symplectic manifold, the mapping $\widetilde{p}: \Omega^{1}(M) \longrightarrow V^{1}(M)$, is an isomorphism, and its restriction on the subspace of the closed 1-forms, it gives an isomorphism to the space symplectic vector fields on $M$. This isomorphism allows us to carry the Lie algebra structure from $V^{1}(M)_{S}$ to the space $Z^{1}(M)$ - the space of closed one-form on the manifold $M$. It is clear, that

$$
i_{[\mid X, Y]} \omega=L_{X}\left(i_{Y} \omega\right)=\mathrm{d}\left(\left(i_{X} \circ i_{Y}\right)(\omega)\right)
$$

which implies that $B^{1}(M)$ - the space of exact 1-forms on the manifold $M$ is a Lie algebra ideal in $Z^{1}(M)$. Moreover:

$$
\left[Z^{1}(M), Z^{1}(M)\right] \subset B^{1}(M)
$$

(see 33 ).

Let us denote by $V^{1}(M)_{H}$ the subalgebra of the Hamiltonian vector fields on the manifold $M$. It is clear that $V^{1}(M)_{H} \subset V^{1}(M)_{S}$. From $\left[Z^{1}(M), Z^{1}(M)\right] \subset$ $B^{1}(M)$ follows that

$$
\left[V^{1}(M)_{S}, V^{1}(M)_{S}\right] \subset V^{1}(M)_{H}
$$

Let $G$ is a Lie group that acts on the manifold $M$, so that for any $g \in G$, the corresponding diffeomorphism $g: M \longrightarrow M$ is a symplectic one: $g^{*}(\omega)=\omega$. In this case, the triple $(M, \omega, G)$ is said to be a symplectic $G$-space. If the action of the group $G$ is transitive, then it is called the homogeneous symplectic $G$-space. 
For a symplectic $G$-space $(M, \omega, G)$, we have the canonical mapping $\sigma$ : $g \longrightarrow V^{1}(M)_{S}$, which assigns to each element $u$ of the Lie algebra of the Lie group $G$, the vector field $\sigma(u)$ corresponding to the one parameter group of diffeomorphisms $\exp (t \cdot u)$.

A symplectic $G$-space $(M, \omega, G)$ is said to be strictly symplectic if for for any $u \in g: \sigma u \in V^{1}(M)_{H}$.

Let $h: C^{\infty}(M) \longrightarrow V^{1}(M)$ be the Hamiltonian mapping, which assigns to each smooth function $f \in C^{\infty}(M)$ the corresponding Hamiltonian vector field $X_{f}$. A mapping $\lambda: g \longrightarrow C^{\infty}(M)$ is called the momentum map, if $h \circ \lambda=\sigma$.

Let us consider the following exact sequence

$$
0 \longrightarrow H^{0}(M, \mathbf{R}) \stackrel{\mu}{\longrightarrow} C^{\infty}(M) \stackrel{h}{\longrightarrow} V^{1}(M)_{S} \stackrel{\eta}{\longrightarrow} H^{1}(M, \mathbf{R}) \longrightarrow 0
$$

The canonical Lie algebra structure on the space $H^{1}(M)$ is trivial, and from $\left[V^{1}(M)_{S}, V^{1}(M)_{S}\right] \subset h\left(C^{\infty}(M)\right)$, follows that the mapping $\eta$ is a Lie algebra homomorphism. In the same manner, the mapping $\mu$ is a Lie algebra homomorphism too. Therefore, it can be stated that the above exact sequence is an exact sequence of Lie algebra homomorphisms. A momentum map $\lambda: g \longrightarrow C^{\infty}(M)$ exists iff Image $(\sigma) \subset h\left(C^{\infty}(M)\right)$, which is equivalent $\eta \circ \sigma=0$. As the composition mapping $\eta \circ \sigma$ is a homomorphism of Lie algebras, and the Lie algebra $H^{1}(M)$ is commutative, we have that $(\eta \circ \sigma)([g, g])=0$. Therefore, the mapping $\eta \circ \sigma \equiv \theta$ induces the quotient mapping

$$
\tilde{\theta}: g /[g, g] \longrightarrow H^{1}(M)
$$

After this, it can be stated that, the necessary and sufficient condition, for existence of the momentum mapping is the equality $\widetilde{\theta}=0$. This condition can be satisfied in the following three cases (see [33]):

Case 1: the Lie algebra $g$ is semisimple $([g, g]=g)$;

Case 2: if the differential form $\omega$ is exact: $\omega=\mathrm{d} \alpha$ and the 1-form $\alpha$ is invariant under the action of the Lie group $G$ : for any $u \in g, L_{\sigma(u)} \alpha=0$; in this case, we have an explicitly defined momentum mapping $\lambda(u)=-\alpha(\sigma(u))$;

Case 3: $H^{1}(M)$ - the first De Rham cohomology space of the manifold $M$, is trivial one.

To investigate the question: is the momentum map

$$
\lambda: g \longrightarrow C^{\infty}(M)
$$

a Lie algebra homomorphism or not? consider the following construction: let the manifold $M$ be strictly symplectic (i.e, image $\left.(\sigma) \subset V^{1}(M)_{H}\right)$. Consider the mapping $m: M \longrightarrow g^{*}$, defined as

$$
m(x)(u)=\lambda(u)(x)
$$


for $x \in M$ and $u \in g$; and a bilinear mapping

$$
c: g \times g \longrightarrow C^{\infty}(M)
$$

defined as

$$
c\left(u_{1}, u_{2}\right)=m\left(\left[u_{1}, u_{2}\right]\right)-\left\{m\left(u_{1}\right), m\left(u_{2}\right)\right\}
$$

The mapping $c$ is obviously antisymmetric; the composition mapping $h \circ c$ is trivial and for any $u, v, w \in g$ we have that

$$
c([u, v], w)+c([v, w], u)+c([w, u], v)=0
$$

The latter equality, together with the antisymmetricity is the condition for the mapping $c$, being a two-dimensional cocycle for the Lie algebra $g$, with values in the ring $C^{\infty}(M)$.

The momentum map $\lambda: g \longrightarrow C^{\infty}(M)$ is a Lie algebra homomorphism if and only if the above defined 2-dimensional cocycle $c$, is trivial (see 33 ).

Now, let us return to the general situation, when the bivector field $p$ on a Poisson manifold $M$, is not necessarily non-degenerated (i.e., the manifold $M$ is not necessarily symplectic one).

Let $\pi$ be the differential system on $M$ derived by the set of the vector fields of the type $X_{f}=\{f, \cdot\}$ for $f \in C^{\infty}(M)$.In other words, for any point $x \in M$, the subspace $\pi(x) \subset \mathrm{T}_{x}(M)$ is defined as

$$
\pi(x)=\left\{u_{\alpha} \in \mathrm{T}_{x}(M) \mid \beta\left(u_{\alpha}\right)=(\alpha \wedge \beta)(p(x)): \alpha, \beta \in \mathrm{T}_{x}^{*}(M)\right\}
$$

The rank of a bivector field at any point $x \in M$ is defined as

$$
\operatorname{Rank}(p)=2 k \Longleftrightarrow \wedge^{k} p(x) \neq 0 \text { and } \wedge^{k+1} p(x)=0
$$

For any function $f \in C^{\infty}(M)$, let

$$
\left\{\Phi_{t}^{f} \mid t \in \mathbf{R}\right\}
$$

be the one-parameter group of diffeomorphisms of the manifold $M$, corresponding to the vector field $X_{f}=\{f, \cdot\}$.

For any two functions $g, h \in C^{\infty}(M)$ and a point $x \in M$, consider the function $\lambda_{x}: \mathbf{R} \longrightarrow \mathbf{R}$ defined as

$$
\lambda_{x}(t)=\left(\left\{g \circ \Phi_{t}^{f}, h \circ \Phi_{t}^{f}\right\}-\{g, h\} \circ \Phi_{t}^{f}\right)(x)
$$

As $\Phi_{t}^{f}, t \in \mathbf{R}$ is a one-parameter group of diffeomorphisms corresponding to some vector field, the element $\Phi_{t}^{f}$ is the identical mapping. Therefore, we have that $\lambda_{x}(0)=0$. Also, it is clear that

$$
\dot{\lambda_{x}}(t)=\left(\left\{X_{f}(g), h\right\}+\left\{g, X_{f}(h)\right\}-X_{f}(\{g, h\})\right)\left(\Phi_{t}^{f}(x)\right)
$$

which is equivalent to

$$
\dot{\lambda_{x}}(t)=\left(\{\{f, g\}, h\}+\{g,\{f, h\}\}-\{f,\{g, h\})\left(\Phi_{t}^{f}(x)\right)\right.
$$


Take into consideration the Jacoby identity for the Poisson bracket $\{\cdot, \cdot\}$, the last equality implies that $\dot{\lambda_{x}}(t)=0$ for any $x \in M$. Hence, we obtain that

$$
\left\{g \circ \Phi_{t}^{f}, h \circ \Phi_{t}^{f}\right\}=\{g, h\} \circ \Phi_{t}^{f}
$$

which is equivalent to

$$
\left(\mathrm{d}\left(g \circ \Phi_{t}^{f}\right) \wedge \mathrm{d}\left(h \circ \Phi_{t}^{f}\right)\right)(p) \circ\left(\Phi_{t}^{f}\right)^{-1}=(\mathrm{d} g \wedge \mathrm{d} h)(p)
$$

The last equality implies that the bivector field $p$ is invariant under the action of the one-parameter group $\Phi_{t}^{f}$ for every smooth function $f \in C^{\infty}(M)$.

It is natural to ask: is the differential system $\pi$ integrable or not?

Note that $\pi$ is an involutive differential system:

$$
\begin{aligned}
& (X, Y) \in \pi \Leftrightarrow\left(X=\sum_{i} \varphi_{i}\left\{f_{i}, \cdot\right\}, Y=\sum_{i} \psi_{i}\left\{g_{i}, \cdot\right\}\right) \Rightarrow \\
& \Rightarrow[X, Y]=\sum_{i}\left(\varphi_{i}\left\{f_{i}, \psi_{i}\right\} \cdot\left\{g_{i}, \cdot\right\}-\psi_{i}\left\{g_{i}, \varphi_{i}\right\}\left\{f_{i}, \cdot\right\}\right)+ \\
& +\sum_{i} \varphi_{i} \psi_{i}\left\{\left\{f_{i}, g_{i}\right\}, \cdot\right\} \in \pi
\end{aligned}
$$

Moreover, the following theorem gives the exact condition for any bivector field $p$, the corresponding differential system be involutive:

Theorem 3 The differential system $\pi$ corresponding to a bivector field $p$ is involutive if and only if

$$
[p, p](x) \in \pi(x) \wedge \pi(x) \wedge \pi(x)
$$

for every point $x \in M$.

Proof. For proof, the following formula is useful: for $\omega \in \Omega^{2}(M), \alpha, \beta \in \Omega^{1}(M)$ and $X, Y \in V^{2}(M)$

$$
\begin{aligned}
& (\omega \wedge \alpha \wedge \beta)(X \wedge Y)= \\
& =\omega(X) \cdot(\alpha \wedge \beta)(Y)+\omega(Y) \cdot(\alpha \wedge \beta)(X)- \\
& -\omega(\tilde{X}(\alpha), \tilde{Y}(\beta))+\omega(\widetilde{X}(\beta), \tilde{Y}(\alpha))
\end{aligned}
$$

Where $\widetilde{X}$ and $\widetilde{Y}$ are the mappings from $\Omega^{1}(M)$ to $V^{1}(M)$ defined by the formula 17. It is sufficient to verify this formula in the case when $\omega=\varphi \wedge \psi$, for 
any $\varphi, \psi \in \Omega^{1}(M)$. In this case we have the following:

$$
\begin{aligned}
& (\varphi \wedge \psi \wedge \alpha \wedge \beta)(X \wedge Y)=(\varphi \wedge \psi)(X) \cdot(\alpha \wedge \beta)(Y)+ \\
& +(\varphi \wedge \alpha)(X) \cdot(\beta \wedge \psi)(Y)+(\varphi \wedge \beta)(X) \cdot(\varphi \wedge \alpha)(Y)+ \\
& +(\psi \wedge \alpha)(X) \cdot(\varphi \wedge \beta)(Y)+(\psi \wedge \beta)(X) \cdot(\alpha \wedge \varphi)(Y)+ \\
& +(\alpha \wedge \beta)(X) \cdot(\varphi \wedge \psi)(Y)= \\
& =\omega(X) \cdot(\alpha \wedge \beta)(Y)+\omega(Y) \cdot(\alpha \wedge \beta)(X)- \\
& -\omega(\widetilde{X}(\alpha), \widetilde{Y}(\beta))+\omega(\widetilde{X}(\beta), \widetilde{Y}(\alpha))
\end{aligned}
$$

The statement of the theorem, translated on the language of a local coordinate system $\left\{x_{1}, \ldots, x_{n}\right\}$ is the following: for each $i, j \in\{1, \ldots, n\}$ the vector field $\left[\widetilde{p}\left(d x_{i}\right), \widetilde{p}\left(d x_{j}\right)\right]$ takes its values in the differential system $\pi$; which is the same as, that $\sigma\left(\left[\widetilde{p}\left(d x_{i}\right), \widetilde{p}\left(d x_{j}\right)\right]\right)=0$ for each $\sigma \in(\pi)^{\perp} \subset \Omega^{1}(M)$.

Using the formula 22 for the Schouten bracket, we obtain:

$$
\begin{aligned}
& \left(\mathrm{d} \sigma \wedge \mathrm{d} x_{i} \wedge \mathrm{d} x_{j}\right)(p \wedge p)= \\
& =(2 \mathrm{~d} \sigma)(p) \cdot\left(\mathrm{d} x_{i} \wedge \mathrm{d} x_{j}\right)(p)-\left(\sigma \wedge \mathrm{d} x_{i} \wedge \mathrm{d} x_{j}\right)([p, p])
\end{aligned}
$$

By using of the formula 28, we obtain:

$$
\begin{aligned}
& \left(d \sigma \wedge \mathrm{d} x_{i} \wedge \mathrm{d} x_{j}\right)(p \wedge p)= \\
& =(2 \mathrm{~d} \sigma)(p) \cdot\left(\mathrm{d} x_{i} \wedge \mathrm{d} x_{j}\right)(p)-(2 \sigma)\left(\widetilde{p}\left(\mathrm{~d} x_{i}\right), \widetilde{p}\left(\mathrm{~d} x_{j}\right)\right)
\end{aligned}
$$

and finally, we have the following:

$$
\left(\sigma \wedge \mathrm{d} x_{i} \wedge \mathrm{d} x_{j}\right)([p, p])=-(2 \sigma)\left(\widetilde{p}\left(\mathrm{~d} x_{i}\right), \widetilde{p}\left(\mathrm{~d} x_{j}\right)\right)
$$

take into consideration the fact that $\sigma \in(\pi)^{\perp}$, the last equality ends the proof of the theorem.

If the rank of a differential system $\pi$ (or, which is the same, the rank of the tensor field $p$ ) is constant, then its integrability follows from the Frobenius's classical theorem; but generally, the differential system $\pi$, is not of a constant rank. Recall, that as it follows from the Hermann's theorem, the necessary and sufficient condition for the integrability of a differential system is the conservation of the rank of the system along the integral paths of this system. This condition is satisfied for the differential system $\pi$, and it follows from the fact 
that the bivector field $p$ is invariant under the action of one-parameter group of the Hamiltonian vector field corresponding to any smooth function on the manifold.

An integral leaf of the differential system $\pi$ is called a symplectic leaf of Poisson structure $p$.

The restriction of the Poisson structure $p$ on any integral leaf of the differential system $\pi$ is non-singular because the rank of this restriction coincides with the dimension of the leaf; hence, such restriction induces a symplectic structure on this leaf.

Let us denote the symplectic form on a symplectic leaf $N$ corresponding to the restriction of the Poisson structure on this leaf by $\omega_{N}$. For $x \in N, u \in$ $\mathrm{T}_{x}(N)$, and $v \in \mathrm{T}_{x}(N)$ we have that $\omega_{N}(u, v)=\{f, g\}(x)$, where $f$ and $g$ are such functions on the manifold $M$ that $u=\{f, \cdot\}(x)$, and $v=\{g, \cdot\}(x)$.

One of the indicators of the singularity of a Poisson structure is the existence of such non-constant smooth function on $M$, which commutes with all smooth functions on $M$, i.e. the center of the Lie algebra of smooth functions, does not coincide to the set of the constant functions. The elements of the center $Z(M)$ are referred as Casimir functions. From the singularity of the Poisson structure $p$ does not follow the existence of a non-constant Casimir function. For instance, if one of the symplectic leaves of the Poisson structure is everywhere dense in the manifold $M$ then a Casimir function can be only constant function. To illustrate this more explicitly, consider the following

Example 1 let $M$ be a two-dimensional symplectic manifold and $p$ be the corresponding non-singular bivector field on $M$. Let $\varphi$ be a non-constant smooth function on $M$. The bivector field $p_{1}=\varphi \cdot p$, is involutive as well as $p$. If the set $\varphi^{-1}(0)$ is not empty, the Poisson structure defined by $p_{1}$, is singular at the points of the set $\varphi^{-1}(0)$, which follows from the relation between the bracket $\{\cdot, \cdot\}_{1}$ defined by the bivector field $p_{1}$ and the bracket $\{\cdot, \cdot\}$ defined by the symplectic structure $p$

$$
\{f, g\}_{1}=\varphi \cdot\{f, g\}
$$

for any $f, g \in C^{\infty}(M)$.

If a function $f \in C^{\infty}(M)$ is a Casimir function, then we have the following

$$
(\varphi \cdot\{f, \cdot\}=0) \Rightarrow\left(\left.\{f, \cdot\}\right|_{M \backslash \varphi^{-1}(0)}=0\right) \Rightarrow(f=\text { const })
$$

If $\varphi$ is such function, that the set $M \backslash \varphi^{-1}(0)$ is everywhere dense set in the manifold $M$ (for example, in the case when the set $\left.\varphi^{-1}(0)\right\}$ consists only one point $\left.x_{0}\right)$, then we have that the function $f$ is constant on the manifold $M$. So, this is an example of the situation when a Poisson structure is singular, but Casimir function can be only constant.

Further, we shall extend (in some sense) the definition of the Poisson bracket on distributions (generalized functions) on a smooth manifold, and be looking for Casimir functions in the set of distributions. 


\subsection{Brief Review of Distributions (Generalized Functions) on Smooth Manifold}

The theory of generalized functions is a common method which allows to manipulate of divergent integrals and series, to differentiate non-smooth functions and perform several such kind of operations over various singular objects. In the following two sections, we use the language of the generalized functions to extend some algebraic notions common for regular Poisson manifolds to the case of singular ones.

For a smooth manifold $M$, let $C_{0}^{\infty}(M)$ be the subalgebra of the algebra $C^{\infty}(M)$, consisting of the functions with compact support. Any linear, continuous functional on the space $C_{0}^{\infty}(M)$ we call a generalized function or distribution on the manifold $M$. The space of all generalized functions on the manifold $M$ we denote further by $\mathcal{D}(M)$. Using the classical notation, the value of a generalized function $\Phi$ on a smooth function with compact support $\varphi$, we denote by $\langle\Phi, \varphi\rangle$.

Let $M$ be a $n$-dimensional oriented manifold with volume form vol $\in \Omega^{n}(M)$. A function $f$, on the manifold $M$, is called a locally integrable function, if for any compact subset $K$ of the manifold $M$ the restriction of the function $f$ on the subset $K$ is integrable under the volume form vol. Such function, defines a functional $[f]: C_{0}^{\infty}(M) \longrightarrow \mathbf{R}$ via the action: for any $\varphi \in C_{0}^{\infty}(M)$ let

$$
[f](\varphi)=\int_{M} f \varphi \cdot \operatorname{vol}
$$

This functional is evidently linear and smooth and subsequently is a generalized function. If we denote by $F(M)$ the space of locally integrable functions on the manifold $M$, it can be stated that there is a mapping $F(M) \longrightarrow \mathcal{D}(M), f \mapsto[f]$. If two functions $f, g \in F(M)$ differ only on a 0 -measure subset of the manifold $M$, then we have that $[f]=[g]$; and inversely: for any two functions $f, g \in$ $F(M)$, if $[f]=[g]$, then they differ only on a 0-measure subset of the manifold $M$ (or in other words: they are equal almost everywhere on the manifold $M$ ). The image of the space $F(M)$ in $\mathcal{D}(M)$ is dense under the topology of weak convergency. Moreover, any generalized function on the manifold $M$ is a weak limit of the sequence of smooth functions on the manifold $M$.

Let us review some algebraic and differential operations over the space of generalized functions on the manifold $M$. These operations are in concordance with the analogical operations on the image of the space $F(M)$, i.e., the mapping $F(M) \longrightarrow \mathcal{D}(M)$ is a homomorphism under these operations. Here is the list of these operations:

Addition: For any two generalized functions $f, g \in \mathcal{D}(M)$ and a smooth function $\varphi \in C_{0}^{\infty}(M)$, let

$$
\langle f+g, \varphi\rangle=\langle f, \varphi\rangle+\langle g, \varphi\rangle
$$

Multiplication: It can be defined a multiplication of a generalized function on a smooth function. For $f \in \mathcal{D}(M), \phi \in C^{\infty}(M)$ and $\psi \in$ 
$C_{0}^{\infty}(M)$, let

$$
\langle\phi \cdot f, \psi\rangle=\langle f, \phi \cdot \psi\rangle
$$

This operation makes the space $\mathcal{D}(M)$ a module over the algebra $C^{\infty}(M)$.

Differentiation: For any vector field $X \in V^{1}(M)$, a generalized function $f \in \mathcal{D}(M)$ and a smooth function $\varphi \in C_{0}^{\infty}(M)$, let

$$
\langle X(f), \varphi\rangle=-\langle f, X(\varphi)\rangle
$$

This operation defines a connection for the pair (see Definition 8) $\left(V^{1}(M), \mathcal{D}(M)\right)$. That is:

for any $f \in \mathcal{D}(M), \varphi \in C^{\infty}(M)$ and $X \in V^{1}(M)$, we have the following

$$
X(\varphi \cdot f)=X(\varphi) \cdot f+\varphi \cdot X(f)
$$

This equality follows from the following series of equalities

$$
\begin{aligned}
& \langle X(\varphi \cdot f), \psi\rangle=-\langle\varphi \cdot f, X(\psi)\rangle= \\
& =-\langle f, \varphi \cdot X(\psi)\rangle= \\
& =\langle f, X(\varphi) \cdot \psi\rangle-\langle f, X(\varphi \cdot \psi)\rangle= \\
& =\langle X(\varphi) \cdot f, \psi\rangle+\langle X(f), \varphi \cdot \psi\rangle= \\
& =\langle X(\varphi) \cdot f, \psi\rangle+\langle\varphi \cdot X(f), \psi\rangle
\end{aligned}
$$

Let $U$ be an open subset of the manifold $M$. Let $C_{0}^{\infty}(M, U)$ be the subspace of the space $C_{0}^{\infty}(M)$, consisting of the smooth functions $\varphi \in$ $C_{0}^{\infty}(M)$ such that: $\varphi=0$ outside of some compact set $K \subset U$. The restriction of a generalized function $f \in \mathcal{D}(M)$ on the open subset $U \in M$ is a functional

$$
\left.f\right|_{U}: C_{0}^{\infty}(M, U) \longrightarrow \mathbf{R}
$$

which is the restriction of the functional $f: C_{0}^{\infty}(M) \longrightarrow \mathbf{R}$ to the subspace $C_{0}^{\infty}(M, U)$.

Push-forward: Let $N$ be another smooth manifold and

$$
F: M \longrightarrow N
$$

be any smooth mapping. For any generalized function $f \in \mathcal{D}(M)$, let $F_{*}(f)$ (push-forward) be a generalized function on the manifold $N$, defined as

$$
F_{*}(f)(\varphi)=f(\varphi \circ F)
$$


for any $\varphi \in C_{0}^{\infty}(M)$.

Using this definition, we can define the right action of the group of diffeomorphisms of the manifold $M$, on the space $\mathcal{D}(M)$ : for any $G \in \operatorname{Diff}(M)$ and $f \in \mathcal{D}(M)$, let

$$
G f=G_{*}^{-1}(f)
$$

A generalized function $f \in \mathcal{D}(M)$ is said to be equal to zero on an open subset $U \subset M$, iff the restriction of $f$ on the subset $U$ equal to zero: $\left.f\right|_{U}=0$. Let the open set $V \subset M$ be the union of all open subset of $M$ on which the generalized function $f$ equal to zero. The complement of the subset $V: M \backslash V$, is called the support of the generalized function $f$, and is denoted by $\operatorname{supp}(f)$.

Let a commutative algebra $A$ is equal to the algebra of smooth functions on the manifold $M$, and $P=\Gamma(\pi)$ be a module of smooth sections of some vector bundle over the manifold $M$. In this case, the vector space (fiber) $\pi^{-1}(x)$ for any point $x \in M$ is canonically isomorphic to the quotient module $P_{x}=P /\left(I_{x} \cdot P\right)$, where $I_{x}$ is a module in the algebra $A$, consisting of the functions equals to 0 , in the point $x$; and $I_{x} \cdot P$ denotes the submodule of the module $P$, generated by the elements of the type $\varphi \cdot s, \varphi \in I_{x}, s \in \Gamma$. Via this isomorphism, the evaluation mapping $P \ni s \mapsto s(x) \in \pi^{-1}(x)$, corresponds to the natural quotient mapping $q_{x}: P \longrightarrow P_{x}$. It is clear that if for some element $s \in P, q_{x}(s)=0, \forall x \in M$, then the element $s$ equal to zero. In general algebraic situation, when $P$ is any module over the algebra $A=C^{\infty}(M)$, it can happen that for some element $s \in P$, we have that $q_{x}(s)=0$ for all points $x \in M$ but the element $s$ is not equal to 0 . Such type of $A$-module, cannot be realized as a module of sections of some vector bundle. They are some kind of non-geometric modules. Now, let us give more strict description of this situation.

A family of elements $\left\{p_{i} \mid i \in I\right\} \subset P$ is called a generated family for the $A$-module $P$, if any element of $P$ can be represented (possibly in more that one manner) as a sum $\sum_{i \in I} a_{i} p_{i}$, with $a_{i} \in A$, where only a finite number of terms in the sum are different from zero. The family $\left\{p_{i} \mid i \in I\right\}$ is called free if it is made of linearly (over the algebra $A$ ) independent elements, and it is a basis for the module $P$ if it is a free generating family; that is: any $s \in P$ can be represented uniquely as a linear combination $\sum_{i \in I} a_{i} p_{i}$. The module $P$ is called free if it admits a basis; and is said to be of finite type if it is finitely generated, i.e., if it admits a generating family with finite number of elements (see [38).

Definition 15 (see [38]) A module over the algebra $A$ is said to be projective if it satisfies the following three equivalent properties:

(pr1) For any epimorphism $\phi: P_{1} \longrightarrow P_{2}$ of A-modules, any homomorphism $f: P \longrightarrow P_{2}$ can be lifted to a homomorphism

$$
\tilde{f}: P \longrightarrow P_{1}
$$

such that $\phi \circ \tilde{f}=f$; 
(pr2) Every epimorphism $f: P_{1} \longrightarrow P$ can be split; i.e., there exists a homomorphism $s: P \longrightarrow P_{1}$ such that $f \circ s=I d_{P}$;

(pr3) The module $P$ is a direct summand of some free module; i.e., there exist a free module $\Gamma$ and a module $P^{\prime}$, such that $\Gamma=P \oplus P^{\prime}$.

The following central statement, provides the criteria for module over the algebra of smooth functions on a smooth manifold to be a module of sections of some vector bundle over this manifold (see [52], [12], [38]).

Proposition 6 Let $M$ be a compact finite dimensional manifold. A $C^{\infty}(M)$ module $P$ is isomorphic to a module of smooth sections of some vector bundle over the manifold $M$, if and only if $P$ is a finite projective module.

For any point $x \in M$, let $\delta_{x} \in \mathcal{D}(M)$ be so-called Dirac function; i.e., it is a linear functional $\delta_{x}: C_{0}^{\infty}(M) \longrightarrow \mathbf{R}$ defined as

$$
\delta_{x}(\varphi)=\varphi(x)
$$

for any $\varphi \in C^{\infty}(M)$. By definition, its derivation is

$$
\delta_{x}^{\prime}(\varphi)=-\delta_{x}\left(\varphi^{\prime}\right)=-\varphi^{\prime}(x)
$$

Lemma 11 For any point $u \in M$, we have that $q_{u}\left(\delta_{x}\right)=0$, where

$$
q_{u}: \mathcal{D}(M) \longrightarrow \mathcal{D}(M) /\left(I_{u} \cdot \mathcal{D}(M)\right)
$$

is the quotient mapping and $I_{u}$ is an ideal in the algebra $C^{\infty}(M)$ consisting of the functions equal to 0 at the point $u$.

Proof. We have to prove that for any point $u \in M$, there exists such generalized function $\eta \in \mathcal{D}(M)$ and a smooth function $\phi \in I_{u}$ that: $\delta_{x}=\phi \cdot \eta$.

Consider two cases: $u \neq x$ and $u=x$.

$\mathbf{u} \neq \mathbf{x}$ : In this case, the function $\phi$, can be any smooth function such that: $\phi(u)=0$ and $\phi(x)=1$. It is clear that $\phi \in I_{u}$. Let us check that the equality, $\delta_{x}=\phi \cdot \delta_{x}$ is true:

for any $\psi \in C_{0}^{\infty}(M)$, we have

$$
\left(\phi \cdot \delta_{x}\right)(\psi)=\delta_{x}(\phi \cdot \psi)=(\phi \cdot \psi)(x)=\phi(x) \cdot \psi(x)=\psi(x)=\delta_{x}(\psi)
$$

therefore, we obtain that when $u \neq x, \delta_{x} \in I_{u}$, which is equivalent to $q_{u}\left(\delta_{x}\right)=0$ in the quotient module $\mathcal{D}(M) /\left(I_{u} \cdot \mathcal{D}(M)\right)$.

$\mathbf{u}=\mathbf{x}$ : In this case, we have that: $\delta_{x}=-\phi \cdot \delta_{x}^{\prime}$, where the function $\phi$ is any smooth function such that $\phi(x)=0$ and $\phi^{\prime}(x)=1$ : 
for any $\psi \in C_{0}^{\infty}(M)$, we have the following

$$
\begin{aligned}
& \left(-\phi \cdot \delta_{x}^{\prime}\right)(\psi)=-\delta_{x}^{\prime}(\phi \cdot \psi)= \\
& =(\phi \cdot \psi)^{\prime}(x)=\phi^{\prime}(x) \cdot \psi(x)+\phi(x) \cdot \psi^{\prime}(x)=\psi(x)=\delta_{x}(\psi)
\end{aligned}
$$

therefore, the Dirac function $\delta_{x}$ is the element of the submodule $I_{x} \cdot \mathcal{D}(M)$, or equivalently $q_{x}\left(\delta_{x}\right)=0$, which finishes the proof.

It follows from this lemma that, for any $x \in M$, the generalized function $\delta_{x}$ is such that $q_{u}\left(\delta_{x}\right)=0$, but the functional $\delta_{x}$ obviously is not equal to zero. This fact implies that the $C^{\infty}(M)$-module $\mathcal{D}(M)$ is not geometric.

\subsection{Poisson Bracket on Generalized Functions and Gen- eralized Casimir Functions}

Let $M$ be a Poisson manifold. The action of the vector fields on the manifold $M$ on the elements of the space $\mathcal{D}(M)$, defines the Poisson bracket of a smooth function $f$ and a generalized function $\Phi$ as:

$$
\{f, \Phi\}=X_{f}(\Phi)
$$

where $X_{f}$ is the Hamiltonian vector field corresponding to the function $f$. The following more detailed definition can be used too: for any $\Phi \in \mathcal{D}(M), f \in$ $C^{\infty}(M)$ and $\psi \in C_{0}^{\infty}(M)$ let

$$
\begin{aligned}
& \langle\{f, \Phi\}, \psi\rangle=\left\langle X_{f}(\Phi), \psi\right\rangle= \\
& =-\left\langle\Phi, X_{f}(\psi)\right\rangle=\langle\Phi,\{\psi, f\}\rangle
\end{aligned}
$$

Hence, it can be stated that we have a connection for the pair $\left(C^{\infty}, \mathcal{D}(M)\right)$, that is: for any $f, g \in C^{\infty}(M)$ and $\Phi \in \mathcal{D}(M)$

$$
\{f, g \cdot \Phi\}=\{f, g\} \cdot \Phi+g \cdot\{f, \Phi\}
$$

which easily follows from the corresponding property for the action of vector fields on generalized function.

If we define $\{\Phi, f\}$ as $-\{f, \Phi\}$ and consider the following operator $\widehat{\Phi}=$ $\{\Phi, \cdot\}: C^{\infty}(M) \longrightarrow \mathcal{D}(M)$, for any fixed $\Phi \in \mathcal{D}(M)$, it turns out that $\widehat{\Phi}$ is a first-order differential operator, with property

$$
\widehat{\Phi}(\phi \psi)=\phi \widehat{\Phi}(\psi)+\psi \widehat{\Phi}(\phi)
$$


The above equality easily follows from

$$
\{\phi \psi, \cdot\}=\phi\{\psi, \cdot\}+\psi\{\phi, \cdot\}
$$

Now, when we have already defined the Poisson bracket of a generalized functionand a smooth function on a Poisson manifold $M$, it can be stated that, if the Poisson structure on the manifold $M$ is such that it is singular but its center coincides with the set of constant functions on $M$, then it has non-constant center in the space of generalized function, on the manifold $M$. That is, there can be found such generalized function $\Phi \in \mathcal{D}(M)$, that $\{\Phi, \psi\}=0$, for every $\psi \in \mathrm{C}^{\infty}(M)$.

In the situation described in the Example 1, the distributions commuting with every smooth function are the Dirac functionals $\delta_{a}$ for $a \in \varphi^{-1}(0)$. In this case, for any $f \in \mathrm{C}^{\infty}(M)$ and $\psi \in C_{0}^{\infty}(M)$, we have the following

$$
\begin{aligned}
& \left\langle\left\{\delta_{a}, f\right\}_{1}, \psi\right\rangle=\left\langle\delta_{a},\{f, \psi\}_{1}\right\rangle= \\
& =\left\langle\delta_{a}, \varphi\{f, \psi\}\right\rangle=\varphi(a)\{f, \psi\}(a)=0
\end{aligned}
$$

Now, we shall describe some general construction to build the distributions "commuting" with all smooth functions on the Poisson manifold $M$.

Let $N$ be a symplectic manifold, i.e., the involutive bivector field correspondent to the Poisson structure on this manifold is non-degenerated. It is the same that the Poisson bracket is defined by some symplectic form $\omega$ as $\{f, g\}=\omega\left(X_{f}, X_{g}\right)$, for $f, g \in \mathrm{C}^{\infty}(M)$, where $X_{f}$ and $X_{g}$ are the Hamiltonian vector fields corresponding to the functions $f$ and $g: \mathrm{d} f=-i_{X_{f}} \omega, \mathrm{d} g=-i_{X_{g}} \omega$.

Let us recall the following formula for the Poisson bracket

$$
\{f, g\} \cdot \omega^{n}=n \cdot \mathrm{d} g \wedge \mathrm{d} f \wedge \omega^{n-1}
$$

where $n=\frac{1}{2} \cdot \operatorname{dim}(M)$ and $\omega^{n}=\underbrace{\omega \wedge \cdots \wedge \omega}_{n \text {-times }}$.

This formula is the result of the following

$$
\begin{aligned}
& \mathrm{d} g \wedge \omega^{n}=0 \quad \Rightarrow \\
& \Rightarrow \quad i_{X_{f}}\left(\mathrm{~d} g \wedge \omega^{n}\right)=\{f, g\} \cdot \omega^{n}-\mathrm{d} g \wedge i_{X_{f}}\left(\omega^{n}\right)=0 \quad \Rightarrow \\
& \Rightarrow \quad\{f, g\} \cdot \omega^{n}=\mathrm{d} g \wedge i_{X_{f}}\left(\omega^{n}\right)
\end{aligned}
$$

Let $M$ be a smooth manifold with Poisson structure defined by a bivector field $p \in V^{2}(M)$ and $N$ be a symplectic leaf in the Poisson manifold $M$. That is, the submanifold $N$ is integral for the distribution defined by the Hamiltonian vector fields and the restriction of the bivector field $p$, on the leaf $N$ is non-degenerated. Therefore $\left.p\right|_{N}$ corresponds to some symplectic form on $N$ which we denote under $\omega_{N}$. Suppose, for convenience, that the manifold $M$ is compact (which implies 
that $\left.\mathrm{C}^{\infty}(\mathrm{M})=C_{0}^{\infty}(M)\right)$ and the submanifold $N$ is closed $(\partial N=0)$. Consider the following generalized function on the manifold $M$ :

$$
\delta_{N}: \mathrm{C}^{\infty}(M) \longrightarrow \mathbf{R}, \quad\left\langle\delta_{N}, \varphi\right\rangle=\left.\int_{N} \varphi\right|_{N} \cdot \omega^{k}
$$

where $\varphi \in \mathrm{C}^{\infty}(M), k=\frac{1}{2} \cdot \operatorname{dim}(M)$ and $\left.\varphi\right|_{N}$ denotes the restriction of the function $\varphi$ to the submanifold $N$.

Proposition 7 For any $\varphi \in C^{\infty}(M)$, we have that $\left\{\delta_{N}, \varphi\right\}=0$

Proof. By definition of the Poisson bracket of a generalized function and a smooth function on a Poisson manifold we have that for any $\varphi, \psi \in \mathrm{C}^{\infty}(M)$

$$
\left\langle\left\{\delta_{N}, \varphi\right\}, \psi\right\rangle=\left\langle\delta_{N},\{\varphi, \psi\}\right\rangle=\left.\int_{N}\{\varphi, \psi\}\right|_{N} \cdot \omega_{N}^{k}
$$

Take into consideration the fact that the Hamiltonian vector fields are tangent to the symplectic leaves, the formula 30 and the Stokes formula, we obtain the following:

$$
\begin{aligned}
& \left.\int_{N}\{\varphi, \psi\}\right|_{N} \cdot \omega_{N}^{k}=\int_{N}\left\{\left.\varphi\right|_{N},\left.\psi\right|_{N}\right\} \cdot \omega_{N}^{k}= \\
& =n \cdot \int_{N} \mathrm{~d} \psi \wedge \mathrm{d} \varphi \wedge \omega_{N}^{k-1}=n \cdot \int_{\partial N} \psi \wedge \mathrm{d} \varphi \wedge \omega_{N}^{k-1}=0
\end{aligned}
$$

\subsection{The Canonical Comlex of a Poisson Manifold and Gen- eralized Casimir Functions}

For a Poisson manifold $M$, with a bivector field $p \in V^{2}(M)$ such that $\{f, g\}=$ $i_{p}(\mathrm{~d} f \wedge \mathrm{d} g)$, Koszul introduced the differential

$$
\delta=i_{p} \circ \mathrm{d}-\mathrm{d} \circ i_{p}: \Omega^{n}(M) \longrightarrow \Omega^{n-1}(M)
$$

(see Section 4.2 for the noncommutative foundation).

Let us enumerate some properties of the operator $\delta$ (see[5]).

The following expression, reveals the relation between the operator $\delta$ and the boundary operator for the Lie algebra homologies:

$$
\begin{aligned}
& \delta\left(f_{0} \mathrm{~d} f_{1} \wedge \cdots \wedge \mathrm{d} f_{k}\right)= \\
& =\sum_{1 \leq i \leq k}(-1)^{i+1}\left\{f_{0}, f_{i}\right\} \mathrm{d} f_{1} \wedge \cdots \wedge \widehat{\mathrm{d} f}_{i} \wedge \cdots \wedge \mathrm{d} f_{k}+ \\
& +\sum_{1 \leq i<j \leq k}(-1)^{i+j} f_{0} \mathrm{~d}\left\{f_{i}, f_{j}\right\} \wedge \cdots \wedge \widehat{\mathrm{d} f}_{i} \wedge \cdots \wedge \widehat{\mathrm{d} f_{j}} \wedge \cdots \wedge \mathrm{d} f_{k}
\end{aligned}
$$


If we denote the Chevalley-Eilenberg complex of the Lie algebra $L=\mathrm{C}^{\infty}(M)$, by $C(L, L)$ it can be stated that $C_{k}(L, L)=L \oplus\left(\wedge^{k} L\right)$ and the differential $\delta: C_{k}(L, L) \longrightarrow C_{k-1}(L, L)$ is given by the formula:

$$
\begin{aligned}
& \delta\left(f_{0} \otimes\left(f_{1} \wedge \cdots \wedge f_{k}\right)\right)= \\
& =\sum_{1 \leq i \leq k}(-1)^{i+1}\left\{f_{0}, f_{i}\right\} \otimes\left(f_{1} \wedge \cdots \wedge \widehat{f}_{i} \wedge \cdots \wedge f_{k}\right)+ \\
& +\sum_{1 \leq i<j \leq k}(-1)^{i+j} f_{0} \otimes\left(\left\{f_{i}, f_{j}\right\} \wedge \cdots \wedge \widehat{f}_{i} \wedge \cdots \wedge \widehat{f}_{j} \wedge \cdots \wedge f_{k}\right)
\end{aligned}
$$

The series of linear mappings: $\pi_{n}: C_{n}(L, L) \longrightarrow \Omega^{n}(M)$, defined as

$$
\pi_{n}\left(f_{0} \otimes\left(f_{1} \wedge \cdots \wedge f_{k}\right)\right)=f_{0} \mathrm{~d} f_{1} \wedge \cdots \wedge \mathrm{d} f_{k}
$$

is a homomorphism of the differential complexes $(C(L, L), \delta)$ and $(\Omega(M), \delta)$. That is, for any $n=0, \ldots, \infty$, we have $\pi_{n} \circ \delta=\delta \circ \pi_{n+1}$.

The differential complex

$$
\cdots \longrightarrow \Omega^{n+1}(M) \stackrel{\delta}{\longrightarrow} \Omega^{n}(M) \longrightarrow \cdots
$$

is called the canonical complex of the Poisson manifold $M$. The homology of this complex is denoted by $H^{\text {can }}(M)$ and called the canonical homology of the Poisson manifold $(M,\{\cdot, \cdot\})$.

Using the formula 31 it is easy to show that: $\mathrm{d} \circ \delta+\delta \circ \mathrm{d}=0$.

If $\alpha$ is a closed differential form on the manifold $M$, from the Koszul's definition of of the operator $\delta$ (see Formula 24) immediately follows that the form $\delta(\alpha)$ is an exact form.

The bivector field $p \in V^{2}(M)$, corresponding to the Poisson structure on the manifold $M$, defines a bilinear pairing for any $k=1, \ldots, \infty$

$$
\wedge^{k}(\hat{p}): \wedge^{k}\left(\mathrm{~T}^{*}(M)\right) \otimes \wedge^{k}\left(\mathrm{~T}^{*}(M)\right) \longrightarrow \mathrm{C}^{\infty} M
$$

by the formula:

$$
\wedge^{k}(\hat{p})(\alpha \otimes \beta)=(\alpha \wedge \beta)\left(\wedge^{k} p\right)
$$

This mapping is $(-1)^{k}$-symmetric.

As in the case of a Riemannian manifold, it can be defined the $\star$ operator in the case of symplectic manifold

$$
\star: \Omega^{k}(M) \longrightarrow \Omega^{2 n-k}(M)
$$

by the formula

$$
\beta \wedge(\star(\alpha))=\wedge^{k}(\hat{p})(\beta, \alpha) \cdot \text { vol }
$$

where $2 n=\operatorname{dim} M ; \quad \alpha, \beta \in \Omega^{k}(M) \quad$ and $\quad \operatorname{vol}=\frac{1}{m !} \cdot \omega^{m}$. 
Remark 2 The noncommutative definition of the $\star$ operator is given in the section 4.7.

The operator $\star$ is involutive: $\star \circ \star=\mathrm{Id}$.

Theorem 4 (see [5]) The relation $\delta=(-1)^{k} \star \circ d \circ \star$ holds on $\Omega^{k}(M)$ for any integer $k \geq 0$.

Corollary 1 (see [5]) For a symplectic manifold, the operator $\star$ defines an isomorphism of the canonical homology $H_{\bullet}^{\text {can }}(M)$ with the de Rham cohomology $H^{2 m-\bullet}(M)$, where $m$ is the dimension of this manifold.

Let $\mathcal{D}_{0}(M)$ be the subspace of the space $\mathcal{D}(M)$ consisting of the generalized function commuting with every smooth function on the manifold $M$;

$H_{0}(M, \delta)$ be the space of the 0-dimensional homologies of the canonical complex of the Poisson manifold $M$;

$H_{0}^{\text {can }}(M)^{*}$ be the space of the linear functionals on the space $H_{0}^{\text {can }}(M)$.

Proposition 8 The spaces $\mathcal{D}_{0}(M)$ and $H_{0}^{\text {can }}(M)^{*}$ are isomorphic.

Proof. As it follows from the definition of the Poisson bracket of a distribution and a smooth function, the space $\mathcal{D}_{0}(M)$ can be defined as

$$
\mathcal{D}_{0}(M)=\left\{\Phi \in \mathcal{D}(M) \quad \mid \quad\langle\Phi,\{f, g\}\rangle=0 \quad \forall f, \forall g \in \mathrm{C}^{\infty} M\right\}
$$

In other words: $\mathcal{D}_{0}(M)=\left\{\mathrm{C}^{\infty} M, \mathrm{C}^{\infty} M\right\}^{\perp}$, where $\left\{\mathrm{C}^{\infty} M, \mathrm{C}^{\infty} M\right\}$ denotes the space of the sums of the type

$$
\sum_{i, j}\left\{\varphi_{i}, \psi_{j}\right\}, \quad \varphi, \psi \in \mathrm{C}^{\infty} M
$$

As it follows from the formula 31 for the canonical coboundary operator

$$
\delta: \Omega(M) \longrightarrow \Omega(M)
$$

its action on the form $\alpha=\sum_{i} \varphi \mathrm{d} \psi \in \Omega^{1}(M)$ is $\delta(\alpha)=\sum_{i}\{\varphi, \psi\}$.

Therefore:

$$
\delta\left(\Omega^{1}(M)\right)=\left\{\mathrm{C}^{\infty} M, \mathrm{C}^{\infty} M\right\}
$$

But, by definition, we have that

$$
H_{0}(M, \delta)=\mathrm{C}^{\infty} M / \delta\left(\Omega^{1}(M)\right)
$$

hence, we obtain that $\delta\left(\Omega^{1}(M)\right)^{\perp}=H_{0}(M, \delta)^{*}$.

Corollary 2 For a compact symplectic manifold $M$ (i.e., the bivector field, corresponding to the Poisson bracket is non-degenerated), the space $\mathcal{D}_{0}(M)$ is one-dimensional and the functional $\left\langle\delta_{\omega}, \varphi\right\rangle=\int_{M} \varphi \cdot \omega^{n}$, where $\varphi \in C^{\infty}(M), \omega$ is a symplectic form and $n=\frac{1}{2} \cdot \operatorname{dim}(M)$, is a basis of the space $\mathcal{D}_{0}(M)$. 
Proof. As $M$ is a symplectic manifold, then the mapping

$$
\star: H_{0}^{\mathrm{can}}(M) \longrightarrow H^{2 m}(M)
$$

where $H^{2 m}(M)$ is the $2 m$-dimensional de Rham cohomology space of the manifold $M$, is isomorphism. As the manifold $M$ is symplectic, it is an oriented manifold; that is: $H^{2 m}(M) \cong \mathbf{R}$.

Let $N$ be a symplectic leaf in the Poisson manifold $M$, and

$$
r: \mathrm{C}^{\infty}(M) \longrightarrow \mathrm{C}^{\infty}(N)
$$

be the restriction mapping. It is clear that $\delta_{N}=r^{*}\left(\delta_{\omega_{N}}\right)$, where

$$
r^{*}: \mathcal{D}(N) \longrightarrow \mathcal{D}(M)
$$

is the dual mapping, and $\omega_{N}$ is the symplectic form on $N$ induced by the restriction of the bivector field on the submanifold $N$. If the mapping $r$ is an epimorphism, then

$$
\operatorname{Image}\left(r^{*}\right)=\left(I_{N}\right)^{\perp}
$$

where $I_{N}$ is the ideal of the functions on $M$ vanishing on the submanifold $N$, and $\left(I_{N}\right)^{\perp}$ is its orthogonal subspace in the space $\mathcal{D}(M)$.

Proposition 9 If a symplectic leaf $N$ in the Poisson manifold $M$ is such that the restriction mapping $r: C^{\infty}(M) \longrightarrow C^{\infty}(N)$ is an epimorphic, then the space $\left(I_{N}\right)^{\perp} \cap \mathcal{D}_{0}(M)$ is one-dimensional and the set $\left\{\delta_{N}\right\}$ gives its basis.

Proof. As the mapping $r$ is a Poisson mapping (i.e., a homomorphism of the Poisson algebras), we have that: $r(\{\varphi, \psi\})=\{r(\varphi), \psi\}$, and therefore: $\left(r^{*}\right)^{-1}\left(\left(I_{N}\right)^{\perp} \cap \mathcal{D}_{0}(M)\right)=\mathcal{D}_{0}(N)$, which is one-dimensional according to the corollary 2 .

\subsection{Poisson Ideal and Reduction of Poisson Algebra.}

Let $A$ be an associative Poisson algebra. That is: $A$ is an associative real or complex algebra and a Lie algebra with a commutator $\{\cdot, \cdot\}: A \times A \longrightarrow A$, such that:

$$
\{a, b \cdot c\}=b \cdot\{a, c\}+\{a, c\} \cdot b
$$

for all $a, b, c \in A$.

Definition $16 A$ subset $I \subset A$ is called a Poisson ideal if $I$ is a two-sided ideal under the multiplication operation in the associative algebra $A$ and is a Lie algebra ideal in the Lie algebra A:

$$
(\forall x \in I, \text { and } \forall a \in A) \quad \Rightarrow \quad(x \cdot a \in I, a \cdot x \in I,\{x, a\} \in I)
$$

If $I$ is a Poisson ideal in the Poisson algebra $A$, then the quotient space $A / I$ is a Poisson algebra too, and the canonical projection mapping $q: A \longrightarrow A / I$ is a homomorphism of Poisson algebras: $q\{a, b\}=\{q(a), q(b)\}$, for all $a, b \in A$. 
If a Poisson ideal $I \subset A$ is such that the quotient algebra $A / I$ is a submanifold algebra, then the algebra $A / I$ can be considered a noncommutative analogue of a Poisson submanifold of a Poisson manifold. In this case, the ideal $I$ will be called the Poisson submanifold ideal.

Definition 17 A Poisson structure on a Poisson algebra $A$ is said to be nondegenerated, iff the Poisson algebra $A$ does not contain any Poisson submanifold ideal besides $\{0\}$ and $A$ itself.

A Poisson submanifold ideal $I$ in a Poisson algebra $A$, will be said to be maximal if $I \neq A$ and the Poisson submanifold ideal $I^{\prime}$, containing the ideal $I$ is only $A$.

Let $A$ and $B$ are associative algebras and $f: A \longrightarrow B$ be their homomorphism. We call the homomorphism $f$ the submanifold mapping, if for any submanifold ideal $I \subset B$, the set $f^{-1}(I)$ is also a submanifold ideal in the algebra $A$.

First of all, let us recall that, for any ideal $I \subset B$, the set $f^{-1}(I)$ is also an ideal in the algebra $A$; and then, from the definition of a noncommutative submanifold, follows that if the homomorphism $f: A \longrightarrow B$ is a submanifold mapping then for any such ideal $I \subset B$, that the following sequence of homomorphisms

$$
0 \longrightarrow \operatorname{Der}_{I}(B)_{0} \longrightarrow \operatorname{Der}_{I}(B) \longrightarrow \operatorname{Der}(B / I) \longrightarrow 0
$$

is short, the following sequence of homomorphisms

$$
0 \longrightarrow \operatorname{Der}_{f^{-1}(I)}(A)_{0} \longrightarrow \operatorname{Der}_{f^{-1}(I)}(A) \longrightarrow \operatorname{Der}\left(A / f^{-1}(I)\right) \longrightarrow 0
$$

is also short.

Lemma 12 If $I \subset A$ is a submanifold ideal, then the canonical projection mapping $q: A \longrightarrow A / I$ is a submanifold mapping.

Proof. Consider any submanifold ideal $I^{\prime} \subset A / I$. It is clear, that the quotient algebra $A / q^{-1}\left(I^{\prime}\right)$ is canonically isomorphic to the quotient algebra $S_{I} / I^{\prime}$, where $S_{I}=A / I$. So, we have the following two exact sequences

$$
0 \longrightarrow \operatorname{Der}_{I}(A)_{0} \longrightarrow \operatorname{Der}_{I}(A) \stackrel{r_{1}}{\longrightarrow} \operatorname{Der}(A / I) \longrightarrow 0
$$

and

$$
0 \longrightarrow \operatorname{Der}_{I^{\prime}}(A / I)_{0} \longrightarrow \operatorname{Der}_{I^{\prime}}(A / I) \stackrel{r_{2}}{\longrightarrow} \operatorname{Der}\left(A / q^{-1}(I)\right) \longrightarrow 0
$$

In this situation, we have to prove that the mapping

$$
r_{3}: \operatorname{Der}_{q^{-1}\left(I^{\prime}\right)}(A) \longrightarrow \operatorname{Der}\left(A / q^{-1}(I)\right)
$$

is an epimorphism.

Let us recall that the space $\operatorname{Der}_{I^{\prime}}(A / I)$ is defined as the space of such derivations of the algebra $A / I$, which carries the ideal $I^{\prime}$ to itself. Therefore, the space 
$r_{1}^{-1}\left(\operatorname{Der}_{I^{\prime}}(A / I)\right)$ is a subspace of $\operatorname{Der}(A)$, consisting of such derivatives of the algebra $A$, which carries the ideals $I$ and $q^{-1}\left(I^{\prime}\right)$ to itself. It is clear that $r_{1}^{-1}\left(\operatorname{Der}_{I^{\prime}}(A / I)\right)$ is a subspace of $\operatorname{Der}_{q^{-1}\left(I^{\prime}\right)}(A)$ and the mapping

$$
r_{2} \circ r_{1}: r_{1}^{-1}\left(\operatorname{Der}_{I^{\prime}}(A / I)\right) \longrightarrow \operatorname{Der}\left(A / q^{-1}(I)\right)
$$

which is an epimorphism, is equal to the restriction of the mapping $r_{3}$ to the subspace $r_{1}^{-1}\left(\operatorname{Der}_{I^{\prime}}(A / I)\right)$.

Theorem 5 (The reduction of Poisson algebra) If $A$ is a Poisson algebra and $I \subset A$ is a maximal Poisson submanifold ideal, then the quotient algebra $Q=A / I$ is non-degenerated Poisson algebra.

Proof. Let $I^{\prime} \subset A / I$ be any Poisson submanifold ideal. As the canonical projection mapping $q: A \longrightarrow A / I$ is a submanifold mapping and is an epimorphism, we have that $q^{-1}\left(I^{\prime}\right)$ is a Poisson submanifold ideal in the algebra $A$ and is not equal to $A$. As $I$ is a maximal Poisson subamanifold ideal, we have that $q^{-1}\left(I^{\prime}\right)=I$, which implies that $I^{\prime}=\{0\}$.

Example 2 Let $M$ be a symplectic manifold and $X$ be any submodule of the module $V^{1}(M)$. Let us denote by $C_{X}^{\infty}(M)$, the subalgebra of the commutative algebra $C^{\infty}(M)$, consisting of such smooth functions $f \in C^{\infty}(M)$ that $u(f)=$ $0, \forall u \in X$ and by $X^{\perp}$, the submodule of $V^{1}(M)$, consisting of such elements $u \in V^{1}(M)$, that $\omega(u, X)=\{0\}$, where $\omega$ is the symplectic form on the manifold $M$.

If the submodule $X^{\perp}$ is involutive (i.e., $\left[X^{\perp}, X^{\perp}\right]=0$ ), then the algebra $C_{X}^{\infty}(M)$ is a Poisson algebra (i.e., a Poisson subalgebra of $C^{\infty}(M)$ ). To check this, consider any two elements $f, g \in C_{X}^{\infty}(M)$. We have

$$
\begin{aligned}
& (X(f)=X(g)=0) \Leftrightarrow(\omega(h(f), X)=\omega(h(g), X)=0) \Leftrightarrow \\
& \Leftrightarrow\left(h(f), h(g) \in X^{\perp}\right) \Rightarrow \quad\left([h(f), h(g)]=h(\{f, g\}) \in X^{\perp}\right) \Rightarrow \\
& \Rightarrow \quad(\omega(h(\{f, g\}), X)=\{0\}) \Rightarrow \quad(X(\{f, g\})=0) \Rightarrow \\
& \Rightarrow \quad\left(f, g \in C_{X}^{\infty}(M)\right)
\end{aligned}
$$

In the case when the submodule $X$ is generated by a symplectic action of some Lie group $G$, the submodule $X^{\perp}$ is involutive:

$$
\begin{gathered}
u \in X \quad \Rightarrow \quad L_{u} \omega=0 \quad \Rightarrow \quad d i_{u} \omega=0 \quad \Rightarrow \\
\Rightarrow \quad x \omega(u, y)-y \omega(u, x)-\omega(u,[x, y])=0
\end{gathered}
$$


which implies that, if $x, y \in X^{\perp}$ then $[x, y] \in X^{\perp}$. Hence, we obtain that in the case of a symplectic action of some Lie group, the algebra of invariant functions under this group, is a Poisson subalgebra of $C^{\infty}(M)$.

A Poisson ideal in the Poisson algebra $C_{X}^{\infty}(M)$ can be constructed by using of a function (if such function exists) $\varphi \in C_{X}^{\infty}(M)$ such that $X^{\perp}(\varphi)=\{0\}$, as the ideal generated by the function $\varphi$. 


\section{References}

[1] J. M. Arms, M. J. Gotay and G. Gennings. (Geometric and Algebraic) Reduction for Singular Momentum Maps. Adv. in Math. 79 (1990) pp 43-103.

[2] I. A. Batalin and G. S. Vilkovisky. Existence Theorem for Gauge Algebra. J. Math. Phys. 26 (1985), pp 172-184.

[3] N. N. Bogoliubov and O. S. Parasiuk. Über die Multiplikation des Kausalfunktionen in der Quantentheorie der Felder. Acta Math. 97 (1952), pp 227-266.

[4] R. Bott. Lectures on Characteristic Classes and Foliations. Lectures on Algebraic and Differential Topology. Lec. Notes in Mathematics, Vol. 279, Springer-Verlag, Berlin, 1972.

[5] Jean-Luc Brylinski. A Differentil Complex for Poisson Manifold. J. Diff. Geom. 28, No.1 (1988), pp 93-114

[6] C. Chevalley and S. Eilenberg. Cohomology Theory of Lie Groups and Lie Algebras. Trans. Amer. Math. Soc. 63. (1948) pp 85-124.

[7] J. F. Conn. Normal Forms for Smooth Poisson Structures. Ann. Math. V. 21. (1985) pp 565-593.

[8] A. Connes. Non-commutative Differential Geometry. IHES 62 (1986), p 257

[9] A. Connes. Non-commutative Differential Geometry. Publ. I.H.E.S. 62 (1986) pp 257-360.

[10] A. Connes. The Action Functional in Non-commutative Geometry. Commun. Math. Phys. 117 (1988) pp 673-683.

[11] A. Connes. Noncommutative Geometry. Academic Press, 1994.

[12] A. Connes. Non-commutative Geometry and Physics, in Gravitation and Quantizations. Les Houches, Session LVII, (Elsevier Science B.V., 1995.)

[13] A. Connes. Noncommutative Geometry and Reality. J. Math. Phys. 36 (1995) pp 6194-6231.

[14] M. Dubois-Violette. Dérivations et Calcul Différentiel non Commutatif. C. R. Acad. Sci. Paris, t. 307, Série1, (1988), pp 403-408

[15] M. Dubois-Violette and P. W. Michor. Connections on Central Bimodules. q-alg/9503020 
[16] M. Dubois-Violette and P. W. Michor. Dérivations et Calcul Différentiel non Commutatif II. C. R. Acad. Sci. Paris, t. 319, Série1, (1994), pp 927-931

[17] R. L. Fernandes. Connections in Poisson Geometry I: Holonomy and Invariants. math.dg/0001129.

[18] V. Ginzburg and A. Golubev. Holonomy on Poisson Manifolds and the Modular Class. math.dg/9812153.

[19] P. A. Griffits. The Differential Geometry of Homogeneous Vector Bundles. Trans. Amer. Math. Soc. V. 109 (1963) pp 1-34.

[20] V. Guillemin and S. Sternberg. Geometric Asymptotics. Amer. Math. Soc., Providence (1977).

[21] V. Guillemin and S. Sternberg. The Moment Map and Collective Motion. Ann. Phys. V. 127, No. 1 (1980) pp 220-253.

[22] V. Guillemin and S. Sternberg. The Metaplectic representation, Weyl Operators and Spectral Theory. J. Func. Anal. V. 42, No 2 (1981) pp 128-225.

[23] V. Guillemin and S. Sternberg. Supersymmetry and Equivariant deRham Theory. Springer-Verlag, New York, 1999.

[24] J. L. Heitsch and S. E. Hurder. Geometry of Foliations. J. Diff. Geom. 20 (1984) pp 291-309.

[25] R. Hermann. The Differential Geometry of Foliations. J. Math. Mech. 11 (1962), pp 303-315.

[26] R. Hermann. Cartan's Geometric Theory of Partial Differential Equations. Adv. Math. (1965), 1:3, pp 265-317.

[27] R. Hermann. Vector Bundles in Mathematical Physics. ibid (1970).

[28] R. Hermann. Physical Aspects of Lie Group Theory. University of Montreal Press, Montreal 1974.

[29] M. Hirsch. Differential Topology. Graduate Text in Mathematics V. 33. New York: Springer-Verlag (1966).

[30] W. V. D. Hodge. The theory and Applications of Harmonic Integrals. 2nd edition - Cambridge: Cambridge University Press (1952).

[31] J. Huebschmann. Poisson Cohomology and Quantization. J. fuer die Reine und Angewandte Mathematik 408 (1990), pp 57-113.

[32] J. Huebschmann. Graded Lie-Rinehart Algebras, Graded Poisson algebras, and BRST Quantization I. The Finitely Generated Case. Heidelberg Preprint (1991). 
[33] N. E. Hurt. Geometric Quantization in Action. Applications of Harmonic Analysis in Quantum Statistical Mechanics and Quantum Field Theory. D. Reidel Publishing Company (1983).

[34] M. V. Karasev and V. P. Maslov. Nonlinear Poisson Brackets. Geometry and Quantization. Monograph. Moscow, "Nauka" (1990).

[35] T. Kimura Generalized Classical BRST Cohomology and Reduction of Poisson Manifolds. hep-th/9207080.

[36] S. L. Koszul. Crochet de Schouten - Nijenhuis et Cohomologie. Elie Cartan et les Mathematiques D'adjourd'hui. Asterisque, hors ser., Soc. Math. France. (1985), pp 257-271.

[37] M. Kuranishi. Lectures on Exterior Differential Systems. Bombay: Tata Inst. Fundamental Res., 1962

[38] G. Landi. An Introduction to Noncommutative Spaces and their Geometry. hep-th/9701078.

[39] J. Leray. Analyse Lagrangienne et Mecanique Quantique. RCP 25 IRMA Strasbourg.

[40] A. Lichnerowicz. Derivations et Cohomologies des Algebres de Lie Attaches d'une Variete Symplectique et une Variete Contact Geometrie Symplectique et Physique Mathematique. Coll. Inter. CNRS (1974).

[41] A. Lichnerowicz. Les varièteès de Poisson et leurs algèbres de Lie associèes. J. Diff. Geom. 12 (1977), pp 253-300.

[42] A. Lichnerowicz. Deformation of Quantification. Lect. Notes in Phys. V. 102 (1979), pp 209-219.

[43] Thierry Masson. Manifolds and Quotient Manifolds in Noncommutative Geometry.

[44] A. Nijenhuis and R. W. Richardson. Cohomology and Deformation in Graded Lie Algebras. Bull. Amer. Math. Soc. 72 (1966), pp 1-29.

[45] G. Rinehart. Differential Forms for General Commutative Algebras. Trans. Amer. Math. Soc. 108 (1963), pp 195-222.

[46] J. A. Schouten. Über Differentialkomitanten Zweier Kontravarianter Grössen. Proc. Nederl. Acad. Wetensh., ser. A., V. 43 (1940), pp 449-452.

[47] L. Schwartz. Analize et Syntése Harmonique Dans les Espaces de Distributions. Can. J. Math. 3 (1951), pp 503-512.

[48] P. Schapira. Théorie des Hyperfonctions. Lect. Notes. Math. (1970), pp 126-157. 
[49] J. Sniatycki and A. Weinstein. Reduction and Quantization for Singular Momentum Mappings. Lett. Math. Phys. 7 (1983), pp 155-161.

[50] J. Stasheff. Homological Reduction of Constrained Poisson Algebras. qalg/9603021.

[51] H. Sussmann. Orbits of Families of Vector Fields and Integrability of Distributions. Trans. Amer. Math. Soc. 180 (1973), pp 171-188.

[52] R. G. Swan. Vector Bundles and Projective Modules. Trans. Am. Math. Soc. 105 (1962) pp 264-277.

[53] I. Vaisman. Lectures on the Geometry of Poisson Manifolds. Progress in Mathematics, Vol. 118, Birkhäuser, Berlin 1994.

[54] I. Vaisman. On the Geometric Quantization of Poisson Manifolds. J. of Math. Physics 32 (1991), pp 3339-3345.

[55] A. M. Vinogradov, I. S. Krasilshchik and V. V. Lychagin. Introduction to the Geometry of Nonlinear Differential Equations. Moscow, "Nauka" (1986).

[56] Frank W. Warner. Foundations of Differentiable Manifolds and Lie Groups. Graduate Texts in Mathematics. Springer-Verlag (1983)

[57] A. Weinstein. The Local Structure of Poisson Manifold. J. Differential Geometry 18 (1983), pp 523-557.

[58] A. Weinstein. The Modular Automorphism Group of a Poisson Manifold. J. Geom. Phys. 23 (1997), pp 379-394 